\title{
Strongly interacting Majorana fermions
}

\author{
Ching-Kai Chiu, D.I. Pikulin, and M. Franz \\ Department of Physics and Astronomy, University of British Columbia, Vancouver, BC, Canada V6T $1 Z 1$ and \\ Quantum Matter Institute, University of British Columbia, Vancouver BC, Canada V6T 1Z4
}

(Dated: March 18, 2015)

\begin{abstract}
Interesting phases of quantum matter often arise when the constituent particles - electrons in solids - interact strongly. Such strongly interacting systems are however quite rare and occur only in extreme environments of low spatial dimension, low temperatures or intense magnetic fields. Here we introduce a new system in which the fundamental electrons interact only weakly but the low energy effective theory is described by strongly interacting Majorana fermions. The system consists of an Abrikosov vortex lattice in the surface of a strong topological insulator and is accessible experimentally using presently available technology. The simplest interactions between the Majorana degrees of freedom exhibit an unusual nonlocal structure that involves four distinct Majorana sites. We formulate simple lattice models with this type of interaction and find exact solutions in certain physically relevant one- and two-dimensional geometries. In other cases we show how our construction allows for the experimental realization of interesting spin models previously only theoretically contemplated.
\end{abstract}

\section{INTRODUCTION}

When fermions partially occupy a band that is flat their kinetic energy is quenched and interactions, even when nominally weak, can have a profound effect on the ground state of the system. This paradigm is realized, with spectacular results, in 2D electron gases in perpendicular magnetic field where the interplay between the flat Landau level band structure and the Coulomb interaction gives rise to fractional quantum Hall effect (FQHE) with all its remarkable phenomenology [1, 2]. More recently, it has been realized that magnetic field is not necessary for the formation of FQHE states: one can obtain these, at least in principle, from lattice models that are tuned so that their conduction band is (nearly) flat and at the same time exhibits a non-zero Chern number making it topologically nontrivial [3 8]. When these conditions are met one can achieve FQHE without magnetic field and there has been considerable interest in such systems recently. In practice, however, it is not clear how a lattice system with a topologically non-trivial flat band could be realized experimentally because the occurrence of a flat band typically requires considerable fine tuning of the overlap integrals which are given in solids by crystal chemistry and this is, in most cases, not continuously tunable. Proposals exist to artificially engineer such systems in optical lattices and dipolar spin systems [9. 10].

In this study we introduce a physical lattice system in which a completely flat band can be obtained by tuning a single parameter. The band is unusual because its fundamental degrees of freedom are Majorana fermions [11-14. In the flat band regime the Hamiltonian is dominated by the interaction term and the system is therefore inherently strongly correlated. Its phenomenology differs substantially from the FQHE paradigm. Interesting phases nevertheless arise and we explore them in some detail.

The specific system we consider is depicted in Fig. 1

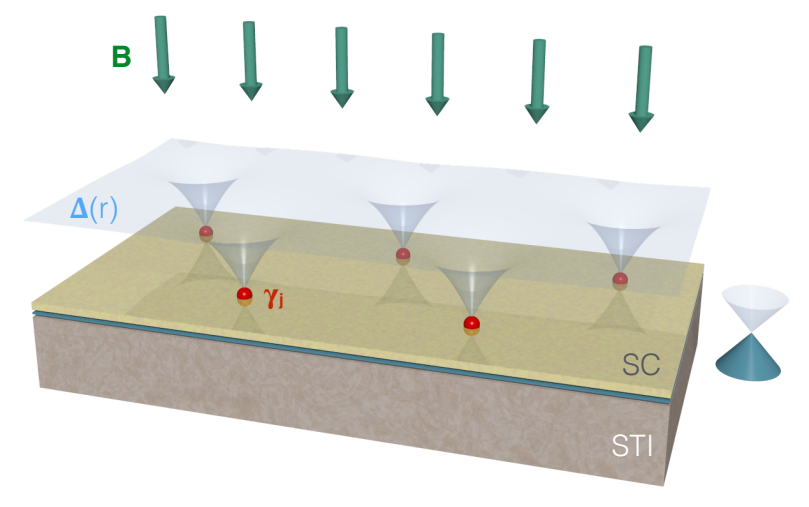

FIG. 1: Schematic depiction of the system based on Fu-Kane model 22]. Superconducting order is induced in the surface of a strong topological insulator (STI) gapping out the protected surface states with the Dirac dispersion. Magnetic field $\mathbf{B}$ is then applied to induce Abrikosov vortices in the SC order parameter $\Delta(\boldsymbol{r})$. Each vortex hosts an unpaired Majorana zero mode $\gamma_{j}$.

and consists of an Abrikosov lattice of vortices in the surface state of a strong topological insulator (STI) that has been made superconducting, either intrinsically as suggested by recent experiments [15, 16], or through the proximity effect with an adjacent ordinary superconductor $17-21]$. Theoretically, the situation is described by the Fu-Kane model 22 which also famously predicts that each vortex in the SC order parameter binds a Majorana zero mode. Tentative experimental evidence for such zero modes has been recently reported in $\mathrm{Bi}_{2} \mathrm{Te}_{3} / \mathrm{NbSe}_{2}$ heterostructures 23.

When two vortices are brought together their Majorana wavefunctions start overlapping and, generically, the zero modes split. In the vortex lattice one thus expects formation of a Majorana band whose bandwidth increases as the lattice becomes denser. This is indeed observed in analytical and numerical calculations [24 30]. However, as we discuss in more detail below, in the spe- 
cial case when the chemical potential $\mu$ of the STI coincides with the Dirac point of the surface state (hereafter referred to as the neutrality point) the band formation can be avoided. This is because the Fu-Kane model at the neutrality exhibits an extra "chiral" symmetry and, as observed by Teo and Kane 31, vortex defects are then in topological class BDI described by an integer (as opposed to $Z_{2}$ valued) invariant. Physically, this means that the total number of exact zero modes in the system is equal to the total vorticity, i.e. the total number $N_{V}$ of vortices present in the system. This is to be contrasted with the $Z_{2}$ classification that applies away from the neutrality point and implies $\left(N_{V} \bmod 2\right)$ exact zero modes.

The above considerations imply that at the neutrality point the chiral symmetry present in the Fu-Kane model prohibits Majorana zero modes from hybridizing, independent of their detailed geometric arrangement. The Majorana band that arises in the vortex lattice therefore remains completely flat. In this situation one may expect interactions to play an important role in determining the collective quantum state of the system. In what follows we explore some of the interesting strongly correlated phases of Majorana fermions that arise in such vortex lattice models. We find that, remarkably, certain strongly interacting models of this type admit exact solutions owing to the presence of an extensive number of conserved quantities. In other cases exact solutions are not available but the Hamiltonians can be mapped onto spin models, some of which have been studied previously and some that appear new.

In Sec. II below we review the general symmetry arguments that indicate the absence of the zero mode hybridization in the Fu-Kane model at neutrality in greater detail. We then outline how this physics arises in a concrete model calculation and use this model in Sec. III to derive the form of the interaction terms and estimate their strength, as well as discuss the effects of small detuning from neutrality on the effective low-energy Hamiltonian of the system. In Sec. IV we proceed to analyze various interacting lattice models with Majorana fermions that can arise in vortex lattices in different oneand two-dimensional geometries. We conclude in Sec. V by discussing prospects for experimental realization and observation of these lattice models in physical systems and we speculate about some novel phases of strongly interacting Majorana matter that can be potentially engineered with help of the tools introduced in this study.

\section{MAJORANA FLAT BANDS IN VORTEX LATTICES}

\section{A. Zero modes in Fu-Kane model}

Fu and Kane 22] envisioned inducing superconductivity in the surface state of a 3D topological insulator by covering it in with a thin film of an ordinary $s$-wave su- perconductor such as $\mathrm{Pb}$ or Nb. Alternately, superconductivity could appear as an intrinsic instability of the surface state [15, 16] or be induced in thin STI flakes through their bulk by placing them on a SC substrate [23. In either case the second-quantized Hamiltonian describing such superconducting STI surface state can be written as

$$
\mathcal{H}=\int d^{2} r \hat{\Psi}_{r}^{\dagger} H_{\mathrm{FK}}(\boldsymbol{r}) \hat{\Psi}_{\boldsymbol{r}}
$$

where $\hat{\Psi}_{\boldsymbol{r}}=\left(c_{\uparrow \boldsymbol{r}}, c_{\downarrow \boldsymbol{r}}, c_{\downarrow \boldsymbol{r}}^{\dagger},-c_{\uparrow \boldsymbol{r}}^{\dagger}\right)^{T}$ is the Nambu spinor and

$$
H_{\mathrm{FK}}(\boldsymbol{r})=\left(\begin{array}{cccc}
-\mu & v p_{-} & \Delta(\boldsymbol{r}) & 0 \\
v p_{+} & -\mu & 0 & \Delta(\boldsymbol{r}) \\
\Delta^{*}(\boldsymbol{r}) & 0 & \mu & -v p_{-} \\
0 & \Delta^{*}(\boldsymbol{r}) & -v p_{+} & \mu
\end{array}\right)
$$

with $p_{ \pm}=p_{x} \pm i p_{y}$ and $\mu$ the chemical potential. The diagonal $2 \times 2$ blocks describe the kinetic energy of the STI surface state (single Dirac fermion with velocity $v$ ) while the off-diagonal blocks encode the SC pair potential.

As the first step we are interested in finding the eigenstates $\Phi_{n}(\boldsymbol{r})$ of $H_{\mathrm{FK}}(\boldsymbol{r})$ in the presence of a single Abrikosov vortex. For a vortex placed at the origin we write

$$
\Delta(\boldsymbol{r})=\Delta_{0}(r) e^{-i(n \varphi+\theta)},
$$

where $\Delta_{0}(r)$ is a real function of the distance, $\varphi$ represents the polar angle and $\theta$ denotes an arbitrary constant phase offset due to other vortices that could be present in the system far away from the origin. Integer $n$ denotes the vorticity. Single-valuedness of the Hamiltonian dictates that $\Delta_{0}(r)$ vanishes at the origin. Energy considerations further show that $\Delta_{0}(r) \sim r^{|n|}$ for small $r$.

To find the zero modes of $H_{\mathrm{FK}}(\boldsymbol{r})$ in the presence of a vortex it is useful to first perform a unitary transformation $\tilde{H}_{\mathrm{FK}}=U H_{\mathrm{FK}} U^{-1}$ with

$$
U=\left(\begin{array}{llll}
1 & 0 & 0 & 0 \\
0 & 0 & 0 & 1 \\
0 & 0 & 1 & 0 \\
0 & 1 & 0 & 0
\end{array}\right)
$$

which brings the Hamiltonian into the following form

$$
\tilde{H}_{\mathrm{FK}}=\left(\begin{array}{cc}
M & D \\
D^{\dagger} & -M
\end{array}\right), \quad D=\left(\begin{array}{cc}
\Delta(\boldsymbol{r}) & p_{-} \\
-p_{+} & \Delta^{*}(\boldsymbol{r})
\end{array}\right) .
$$

and $M=\operatorname{diag}(-\mu, \mu)$. The transformed Hamiltonian acts on the modified Nambu spinor $\hat{\Psi}_{r}=$ $\left(c_{\uparrow \boldsymbol{r}},-c_{\uparrow \boldsymbol{r}}^{\dagger}, c_{\downarrow \boldsymbol{r}}^{\dagger}, c_{\downarrow \boldsymbol{r}}\right)^{T}$. Passing into the polar coordinates and making use of the identity $p_{ \pm}=e^{ \pm i \varphi}\left(-i \partial_{r} \pm r^{-1} \partial_{\varphi}\right)$ we may write

$$
D=\left(\begin{array}{cc}
e^{-i(n \varphi+\theta)} \Delta_{0}(r) & e^{-i \varphi}\left(-i \partial_{r}-\frac{\partial_{\varphi}}{r}\right) \\
-e^{i \varphi}\left(-i \partial_{r}+\frac{\partial_{\varphi}}{r}\right) & e^{i(n \varphi+\theta)} \Delta_{0}(r)
\end{array}\right)
$$


where we have set $v=\hbar=1$. We now temporarily focus on the neutrality point where $M=0$ and the Hamiltonian (5) is purely off-diagonal. When looking for the zero modes the off-diagonal form has a distinct advantage: the zero modes necessarily have the spinor structure $(\psi(\boldsymbol{r}), 0)^{T}$ and $(0, \chi(\boldsymbol{r}))^{T}$ where $\psi(\boldsymbol{r})$ and $\chi(\boldsymbol{r})$ are two-component zero modes of $D^{\dagger}$ and $D$ respectively. For a singly quantized vortex $(n=1)$ it is easy to show that there exists a normalizable zero mode of $D$ of the form

$$
\chi_{0}(\boldsymbol{r})=\frac{1}{\sqrt{2}}\left(\begin{array}{c}
e^{-i(\theta / 2-\pi / 4)} \\
e^{i(\theta / 2-\pi / 4)}
\end{array}\right) f_{0}(r),
$$

with

$$
f_{0}(r)=A e^{-\int_{0}^{r} \Delta_{0}\left(r^{\prime}\right) d r^{\prime}},
$$

while $D^{\dagger}$ does not have a normalizable zero mode. The field operator of the zero mode reads

$$
\gamma=\frac{1}{\sqrt{2}} \int d^{2} r\left[e^{i(\theta / 2-\pi / 4)} c_{\boldsymbol{r} \downarrow}+e^{-i(\theta / 2-\pi / 4)} c_{\boldsymbol{r} \downarrow}^{\dagger}\right] f_{0}(r) .
$$

As expected, the zero mode represents a Majorana particle, $\gamma^{\dagger}=\gamma$. For $\mu \neq 0$ the structure of the zero mode wavefunction becomes slightly more complicated [25]; in addition to the exponential decay it exhibits an oscillatory behavior $\sim \sin k r$ where $k$ is a wavevector close to the Fermi wavevector $k_{F}=\mu / v$.

When multiple well-separated vortices are present in the system then each will harbor a Majorana zero mode. Their respective creation operators $\gamma_{j}$ satisfy the anticommutation algebra [11,14]

$$
\left\{\gamma_{i}, \gamma_{j}\right\}=2 \delta_{i j}, \quad \gamma_{i}^{\dagger}=\gamma_{i},
$$

characteristic of Majorana fermions. The latter follows directly from Eq. (9) generalized to multiple vortices and the canonical anticommutation relations for the electron operators $c_{\boldsymbol{r} \sigma}$. The expected non-Abelian exchange statistics of vortices containing Majorana zero modes [32, 33] becomes apparent when one considers adiabatic exchange of two such vortices. In what follows we shall deal with vortices pinned at fixed positions and their nonAbelian properties will therefore not play an essential role in our considerations.

\section{B. Symmetry considerations}

In the presence of multiple vortices that are closely spaced the fate of the zero modes associated with a single isolated vortex will depend on the symmetries of the underlying Hamiltonian, as discussed in detail by Teo and Kane [31. We now briefly review their analysis as relevant to the Hamiltonian (2). To facilitate the discussion we rewrite the latter in a more compact notation

$$
H_{\mathrm{FK}}=\tau^{z}(\boldsymbol{p} \cdot \boldsymbol{\sigma}-\mu)+\tau^{x} \Delta_{1}+\tau^{y} \Delta_{2}
$$

where $\Delta=\Delta_{1}+i \Delta_{2}$ and $\boldsymbol{\sigma}, \boldsymbol{\tau}$ are Pauli matrices in spin and Nambu spaces, respectively. The Hamiltonian (11) respects the particle-hole symmetry generated by $\Xi=\sigma^{y} \tau^{y} K\left(\Xi^{2}=+1, K\right.$ denotes complex conjugation $)$ and, for a purely real gap function $\Delta$, also the physical time reversal symmetry $\Theta=i \sigma^{y} K\left(\Theta^{2}=-1\right)$. In the presence of vortices $\Delta$ becomes complex and the time reversal symmetry is broken. Fu-Kane model with vortices therefore defines symmetry class D in the AltlandZirnbauer classification which according to Ref. 31 implies a $Z_{2}$ classification for the zero modes associated with point defects such as vortices. Physically, this means that a system with total vorticity $N_{V}$ will have $\left(N_{V} \bmod 2\right)$ exact zero modes, in accord with the expectation that any even number of Majorana zero modes will generically hybridize and form complex fermions at non-zero energies.

However, in the special case when $\mu=0$, Hamiltonian (11) respects a fictitious time reversal symmetry with $\Theta=\sigma^{x} \tau^{x} K\left(\tilde{\Theta}^{2}=+1\right)$, even in the presence of vortices. At the neutrality point, the two symmetries $\Xi$ and $\tilde{\Theta}$ define a BDI class with chiral symmetry $\Pi=\Xi \tilde{\Theta}=\sigma^{z} \tau^{z}$. This, according to Ref. 31implies an integer classification of zero modes associated with point defects. A system with total vorticity $N_{V}$ will thus exhibit $N_{V}$ exact zero modes, irrespective of the details such as the geometric arrangement of the individual vortices. Below we illustrate how this interesting behavior emerges in a concrete model calculation.

We remark that Fu-Kane model at the neutrality point coincides with the Jackiw-Rossi model [34] well known in particle physics, where the $\mu=0$ condition is enforced by Lorentz invariance. An index theorem for Dirac fermions applied to this model [35] is known to connect the total vorticity with the number of protected fermionic zero modes. This property of the Fu-Kane model has been previously noted in Ref. [25].

\section{Zero mode hybridization in a vortex lattice}

We now study the zero mode hybridization using the microscopic wavefunctions obtained above in subsection IIA. To begin consider two vortices located at points $\boldsymbol{R}_{1}$ and $\boldsymbol{R}_{2}$, such that $\left|\boldsymbol{R}_{1}-\boldsymbol{R}_{2}\right| \gg \xi$. The two-vortex Hamiltonian $H_{\mathrm{FK}}^{(2)}$ still has the structure displayed in Eq. (5) except that $\Delta(\boldsymbol{r})$ now encodes vortices at $\boldsymbol{R}_{1}$ and $\boldsymbol{R}_{2}$. We can seek its low-energy eigenstates in the basis spanned by the zero mode wavefunctions $\Phi_{1}(\boldsymbol{r})=\left(0, \chi_{0}\left(\boldsymbol{r}-\boldsymbol{R}_{1}\right)\right)^{T}$ and $\Phi_{2}(\boldsymbol{r})=\left(0, \chi_{0}\left(\boldsymbol{r}-\boldsymbol{R}_{2}\right)\right)^{T}$. If we denote the two Majorana operators as $\gamma_{1}$ and $\gamma_{2}$ then the zero mode splitting comes from the term $i t_{12} \gamma_{1} \gamma_{2}$ with the overlap integral $i t_{12}=\left\langle\Phi_{1}\left|H_{\mathrm{FK}}^{(2)}\right| \Phi_{2}\right\rangle$. At the neutrality point the matrix element $t_{12}$ trivially evaluates to zero because $\left|\Phi_{1}\right\rangle$ is orthogonal to $H_{\mathrm{FK}}^{(2)}\left|\Phi_{2}\right\rangle$ for arbitrary positions $\boldsymbol{R}_{1}$ and $\boldsymbol{R}_{2}$. The zero modes therefore remain exact as expected on the basis of the symmetry argument presented above. 
Away from the neutrality point we find 29

$$
\begin{aligned}
i t_{12} & =\int d^{2} r \chi_{0}^{\dagger}\left(\boldsymbol{r}-\boldsymbol{R}_{1}\right)(-M) \chi_{0}\left(\boldsymbol{r}-\boldsymbol{R}_{2}\right) \\
& =i \mu \sin \left(\frac{\theta_{1}-\theta_{2}}{2}\right) F_{12}
\end{aligned}
$$

with $F_{12}=\int d^{2} r f_{0}\left(\boldsymbol{r}-\boldsymbol{R}_{1}\right) f_{0}\left(\boldsymbol{r}-\boldsymbol{R}_{2}\right)$; the overlap is proportional to $\mu$ and is generally nonzero.

If there are many vortices in the system then the overlap integrals remain zero at the neutrality point and are given by a generalization of Eq. 12 when $\mu \neq 0$. A system of many vortices in a superconductor (or a charged superfluid) is only stable in the presence of an externally applied magnetic field $\boldsymbol{B}$ [42. To describe a realistic vortex lattice we must therefore include magnetic field by performing a minimal substitution $\boldsymbol{p} \rightarrow \boldsymbol{p}-\tau^{z}(e / c) \boldsymbol{A}$ in the Hamiltonian (11). One can show that the presence of $\boldsymbol{A}$ does not qualitatively change the zero mode wavefunction (7) associated with an individual vortex. However, the phase difference $\left(\theta_{1}-\theta_{2}\right) / 2$ in the overlap integral Eq. 12 must be replaced by its gauge invariant generalization

$$
\omega_{12}=\int_{\boldsymbol{r}_{1}}^{\boldsymbol{r}_{2}}\left(\frac{1}{2} \nabla \theta-\frac{e}{c} \boldsymbol{A}\right) \cdot d \mathbf{l}
$$

where the integral is taken along the straight line between vortex positions $\boldsymbol{r}_{1}$ and $\boldsymbol{r}_{2}$. This result can be obtained by an explicit calculation but also follows from a simple general argument: because the overlap amplitudes $\left|t_{i j}\right|$ are potentially measurable physical quantities they cannot depend on an arbitrarily chosen gauge. Some details of how one evaluates the gauge invariant phases 113 in the vortex lattice geometry are provided in Appendix A.

The low-energy effective Hamiltonian describing the Majorana zero modes in a vortex lattice can thus be written as

$$
\mathcal{H}_{\mathrm{kin}}=\sum_{i, j} t_{i j} s_{i j} \gamma_{i} \gamma_{j}
$$

Here we use a notation introduced in Ref. 36 where $t_{i j}$ is a real symmetric matrix representing the hopping strength while $s_{i j}=e^{i \phi_{i j}}= \pm i$ are the $\mathrm{Z}_{2}$ gauge factors. The sign ambiguity arises from the fact that one can perform a local $Z_{2}$ gauge transformation $\gamma_{j} \rightarrow-\gamma_{j}$ without affecting the zero mode commutation algebra 10 . A product of $s_{i j}$ factors along a closed trajectory is however gauge invariant and physically observable. It represents a $Z_{2}$ gauge flux and should be thought of as analogous to the magnetic flux expressed through Peierls factors in lattice models of charged particles. In the vortex lattice for a general polygon formed by $n$ vortices the total phase is given by [36]

$$
\sum_{\text {polygon }} \phi_{i j}=\frac{\pi}{2}(n-2)
$$

In the context of Eqs. 12 13) the $\mathrm{Z}_{2}$ gauge factors arise from the fact that half of the phase difference enters the overlap integral $\sqrt{12}$ and the $\sin \omega_{i j}$ function is thus not single valued in the presence of vortices. The physics of the associated branch cuts and how they give rise to the $\mathrm{Z}_{2}$ gauge factors is further explained in Appendix A

We note that according to Eq. (15) for both triangular and square vortex lattices if $t_{i j}$ are non-zero, Majorana fermions move in a background of non-trivial $\mathrm{Z}_{2}$ flux. This makes even the non-interacting problem interesting and leads to the rich physics of "nucleated" topological phases, explored in previous studies [37, 38.

As already noted, for $\mu \neq 0$ the Majorana wavefunctions exhibit Friedel-like oscillations with lengthscale set by $k_{F}=\mu / v$. When intervortex distance $d$ is such that $k_{F} d \gtrsim 1$ then this leads to an oscillatory behavior of the overlaps $t_{i j}$ with the distance. Such oscillations in combination with disorder in vortex positions have been studied and shown to produce interesting effects [26, 40. In this study we focus on the regime $k_{F}^{-1}>d \gtrsim \xi$ where the oscillatory behavior can be neglected. Oscillations in this regime have no effect on the hoppings between near neighbors and are damped out by the exponential decay of the wavefunctions on longer distances. As we will show in the next Section it is precisely this regime where the interactions tend to dominate over the kinetic energy and this is also where our interest lies.

\section{INTERACTION EFFECTS}

\section{A. General considerations}

We showed in the previous Section that by tuning a single system parameter in the Fu-Kane model (the global chemical potential $\mu$ ) one can eliminate the hybridization between the Majorana zero modes bound to individual vortices. We demonstrated how this occurs in a specific microscopic model but we emphasize that this effect only depends on the system symmetries and not on the microscopic details.

At the neutrality point, therefore, the Majorana band associated with an arbitrary vortex lattice will be completely flat and the manybody ground state will exhibit $2^{N_{V} / 2-1}$-fold degeneracy under the conservation of fermionic parity. At the non-interacting level this degeneracy is robust to any symmetry-preserving perturbation. A question that naturally arises is what physical effects (if any) are likely to remove this extensive ground state degeneracy in a physical system. There are essentially two possibilities: (i) symmetry breaking disorder and (ii) interactions. It is clear that local fluctuations in the chemical potential $\mu$, if allowed, will generate random hoppings $t_{i j}$ between nearby Majorana zero modes and these will in turn remove the ground state degeneracy. This is because non-zero fluctuating $\mu$ breaks the fictitious time reversal symmetry $\tilde{\Theta}$ of the Fu-Kane Hamiltonian at neutrality returning its zero mode classification 
back to class D. Majorana fermion systems with random hoppings have been previously considered in a number of studies [26, 39, 41] .

In this work we focus on the interactions whose effects are much less well understood. Accordingly, we shall consider systems in which the interaction strength is much larger than any perturbation arising from the disorder effects. We will show that conditions under which such an assumption can be justified can indeed occur in physical systems. Specifically, we consider four-fermion terms that arise from Coulomb or possibly other interactions present in the underlying solid state system. Such interactions are generated even when both particle-hole symmetry $\Xi$ and the fictitious time reversal symmetry $\tilde{\Theta}$ are respected. This allows for a genuinely strongly correlated regime in which the physics is completely dominated by interactions and the kinetic energy is quenched.

Under these assumptions the leading perturbation to the degenerate manifold of Majorana zero modes will arise from electron-electron interactions that are necessarily present in the underlying solid. If we denote by $\gamma_{j}$ the annihilation operator of the Majorana zero mode belonging to the $j$-th vortex then the simplest interaction term that can be constructed has the form

$$
\mathcal{H}_{\text {int }}=\sum_{i j k l} g_{i j k l} \gamma_{i} \gamma_{j} \gamma_{k} \gamma_{l}
$$

where $g_{i j k l}$ are real constants representing the interaction strength. The reality of $g_{i j k l}$ follows from the requirement that $\mathcal{H}_{\text {int }}$ be hermitian. Furthermore, since the Majorana operators obey the anticommutation algebra (10) only the part of $g_{i j k l}$ that is antisymmetric in all indices contributes to $\mathcal{H}_{\text {int }}$. We note specifically that according to Eq. $10 p \gamma_{i}^{\dagger} \gamma_{i}=\gamma_{i} \gamma_{i}=1$ and the terms in $\mathcal{H}_{\text {int }}$ with two identical indices reduce to fermion hoppings, e.g. $g_{i i k l} \gamma_{i} \gamma_{i} \gamma_{k} \gamma_{l}=g_{i i k l} \gamma_{k} \gamma_{l}$. However, such terms are not hermitian and one can show that since $g_{i i k l}=g_{i i l k}$ they identically vanish. The simplest interaction term thus involves Majoranas located at four distinct vortices. Such a non-local interaction may be expected to give rise to unusual physical properties.

The expression in Eq. (16) is cumbersome because for every group of four vortices it contains 24 distinct permutations of the $\gamma$ operators. It is thus preferable to rewrite $\mathcal{H}_{\text {int }}$ as a sum over all distinct groups of four vortices in each of which we define a specific ordering of $\gamma$ 's. For example for the group $\left(\gamma_{1}, \gamma_{2}, \gamma_{3}, \gamma_{4}\right)$ we write the interaction term as

$$
\mathcal{H}_{\text {int }}^{1234}=g \gamma_{1} \gamma_{2} \gamma_{3} \gamma_{4}
$$

and similarly for other groups with $\gamma$ 's organized in order of increasing index $j$. The interaction term $\mathcal{H}_{\text {int }}^{1234}$ is allowed to introduce in the Hamiltonian since $\mathcal{H}_{\text {int }}^{1234}$ in Majorana operator basis automatically preserves particlehole symmetry and is invariant under time reversal operation $\gamma_{j} \rightarrow \gamma_{j}$ and $i \rightarrow-i$.

In the next subsection we shall discuss the microscopic origin and the strength of coupling constants $g$. As we shall see the coupling strength $g$ depends on the zero mode wavefunction overlaps as well as the detailed form of the interaction potential $V(\boldsymbol{r})$. For our present purposes it will suffice to note that since the Majorana wavefunctions decay exponentially outside the vortex core, the largest $g$ will occur for those groups of four vortices that are packed closest together. In the following we shall often consider examples of lattice systems in which we retain only such dominant interactions and neglect all $g$ 's associated with groups of vortices that are more spread out since they are smaller by factors $\sim e^{-d / \xi}$ where $d$ is the intervortex distance and $\xi$ the SC coherence length. For instance in the square vortex lattice we shall retain $g_{\square}$ associated with an elementary square plaquette and neglect all other couplings.

\section{B. Microscopic origin of the interaction terms}

Suppose we have solved the single-electron problem in the presence of $N$ vortices. We thus have the complete set of eigenfunctions $\Phi_{n}(\boldsymbol{r})$ and eigenenergies $E_{n}$ of $H_{\mathrm{FK}}^{(N)}$. The second quantized Hamiltonian (1) can then be written in a diagonal form $\mathcal{H}=\sum_{n}^{\prime} E_{n} \psi_{n}^{\dagger} \hat{\psi}_{n}+E_{g}$ where

$$
\hat{\psi}_{n}=\int d^{2} r \Phi_{n}^{\dagger}(\boldsymbol{r}) \hat{\Psi}_{\boldsymbol{r}}
$$

are the eigenmode operators. The sum over $n$ is restricted to the positive energy eigenvalues and $E_{g}$ is a constant representing the ground state energy. At the neutrality point, according to our preceding discussion, $N$ of the $\hat{\psi}_{n}$ eigenmodes coincide with the exact zero modes belonging to the individual vortex cores. We denote these $\gamma_{j}$ with $j=1 \ldots N$.

The Coulomb interaction, appropriately screened, can be written as

$$
U=\frac{1}{2} \iint d^{2} r d^{2} r^{\prime} \hat{\rho}(\boldsymbol{r}) V\left(\boldsymbol{r}-\boldsymbol{r}^{\prime}\right) \hat{\rho}\left(\boldsymbol{r}^{\prime}\right),
$$

where $V(\boldsymbol{r})$ is the interaction potential and $\hat{\rho}(\boldsymbol{r})=c_{\sigma \boldsymbol{r}}^{\dagger} c_{\sigma \boldsymbol{r}}$ is the electron charge density operator. The latter can be expressed in terms of modified Nambu spinors as $\hat{\rho}(\boldsymbol{r})=\hat{\Psi}_{\boldsymbol{r}}^{\dagger} O_{\rho} \hat{\Psi}_{\boldsymbol{r}}$ with $O_{\rho}=\frac{1}{2} \operatorname{diag}(1,-1,-1,1)$. Next, by exploiting the completeness of the eigenstates $\Phi_{n}(\boldsymbol{r})$ we can invert Eq. (18) to obtain

$$
\hat{\Psi}_{\boldsymbol{r}}=\sum_{n} \Phi_{n}(\boldsymbol{r}) \hat{\psi}_{n}
$$

and express the charge density in terms of the eigenmode operators as

$$
\hat{\rho}(\boldsymbol{r})=\sum_{n, m}\left[\Phi_{n}^{\dagger}(\boldsymbol{r}) O_{\rho} \Phi_{m}(\boldsymbol{r})\right] \hat{\psi}_{n}^{\dagger} \hat{\psi}_{m} .
$$

Substituting this result into Eq. 19 and projecting onto the zero mode subspace we arrive at the interaction be- 
tween Majorana modes of the form

$$
U_{0}=\frac{1}{2} \sum_{i j k l} \gamma_{i} \gamma_{j} \gamma_{k} \gamma_{l} \iint d^{2} r d^{2} r^{\prime} \rho_{i j}(\boldsymbol{r}) V\left(\boldsymbol{r}-\boldsymbol{r}^{\prime}\right) \rho_{k l}\left(\boldsymbol{r}^{\prime}\right),
$$

where

$$
\rho_{i j}(\boldsymbol{r})=\left[\Phi_{i}^{\dagger}(\boldsymbol{r}) O_{\rho} \Phi_{j}(\boldsymbol{r})\right] .
$$

Comparing Eqs. 222) and (16) we may read off an expression for $g_{i j k l}$,

$$
g_{i j k l}=\frac{1}{2} \iint d r^{2} d r^{\prime 2} \rho_{i j}(\boldsymbol{r}) V\left(\boldsymbol{r}-\boldsymbol{r}^{\prime}\right) \rho_{k l}\left(\boldsymbol{r}^{\prime}\right) .
$$

At the neutrality point we can use Eq. (7) to write

$$
\rho_{i j}(\boldsymbol{r})=-\frac{i}{2} \sin \left(\frac{\theta_{i}-\theta_{j}}{2}\right) f_{0}\left(\boldsymbol{r}-\boldsymbol{R}_{i}\right) f_{0}\left(\boldsymbol{r}-\boldsymbol{R}_{j}\right) .
$$

In the vortex lattice when magnetic field is present the phase difference is to be replaced by $\omega_{i j}$ defined in Eq. (13). Noting the antisymmetry $\rho_{i j}(\boldsymbol{r})=-\rho_{j i}(\boldsymbol{r})$, the expression for the interaction parameter $g$ defined in Eq. (17) for every such group of four vortices can be written as

$$
g=\epsilon^{i j k l} g_{i j k l}
$$

where $\epsilon^{i j k l}$ is the totally antisymmetric tensor. This can be further simplified, for the group of four Majoranas $\gamma_{1} \ldots \gamma_{4}$, as

$$
g=8\left(g_{1234}+g_{4123}-g_{1324}\right) .
$$

The three distinct terms can now be evaluated with the help of Eq. 25).

\section{Estimate of the interaction strength}

Since according to our analysis in Sec. II the hopping amplitudes between Majorana fermions $t_{i j}$ can be tuned to zero by adjusting the chemical potential the system will be in the strong interaction regime for any non-zero value of $g$. In practice, of course, we need $g$ sufficiently large to be able to observe the interaction effects in a sample with realistic levels of disorder and at nonzero temperature $T$. We thus require an estimate of $g$ relevant to a realistic situation. For concreteness, we consider the vortex lattice in the $\mathrm{Bi}_{2} \mathrm{Te}_{3} / \mathrm{NbSe}_{2}$ heterostructure discussed in Ref. 23 .

In this situation we expect the Coulomb interaction to be well screened so that it is essentially point-like on the scale set by the $\mathrm{SC}$ coherence length $\xi$, i.e. $V(\boldsymbol{r}) \simeq$ $V_{0} \delta(\boldsymbol{r})$. The expression (24) for the coupling constant simplifies, becoming

$$
g_{i j k l}=\frac{1}{2} V_{0} \int d r^{2} \rho_{i j}(\boldsymbol{r}) \rho_{k l}(\boldsymbol{r}) .
$$

Evaluation of the coupling constant in this limit thus involves an estimate of $V_{0}$, calculation of the overlap integral implied by Eq. (28), and a determination of the geometric prefactors coming from the phases $\theta_{i}$ indicated in Eq. 25). We begin with the latter as the phases are determined purely by the vortex lattice geometry. We first consider an elementary square in an infinite periodic square vortex lattice. The phase difference structure is discussed in Appendix A and is consistent with the one obtained in [36]. We then obtain $g_{\square}=-2 V_{0} F_{1234}$ where

$$
F_{1234}=\int d^{2} r \Pi_{j=1}^{4} f_{0}\left(\boldsymbol{r}-\boldsymbol{R}_{j}\right) .
$$

For a linear 1D arrangement of the four vortices one similarly obtains $g_{--}=-V_{0} F_{1234}$.

To estimate $F_{1234}$ we must adopt some specific form for the radial part $f_{0}(\boldsymbol{r})$ of the Majorana wavefunction, which in turn depends on the order parameter profile $\Delta(\boldsymbol{r})$ near the vortex through Eq. (8). In the vicinity of a singly quantized vortex the latter is well approximated by 42

$$
\Delta(\boldsymbol{r})=\Delta_{0} \tanh (r / \xi),
$$

with $\Delta_{0}$ the asymptotic gap value far from the vortex. To facilitate analytical treatment we further expand Eq. (30) at small distances as $\Delta(\boldsymbol{r}) \approx \Delta_{0}(r / \xi)$, which then leads to a normalized wavefunction

$$
f_{0}(\boldsymbol{r}) \simeq \frac{1}{\pi \xi} e^{-r^{2} / 2 \pi \xi^{2}},
$$

where we employed the BCS definition of the coherence length $\xi=v / \pi \Delta_{0}$. The above approximation is valid for $r \lesssim \xi$; for larger radii $f_{0}(\boldsymbol{r})$ crosses over to a simple exponential dependence $\sim e^{-r / \xi}$. The advantage of the approximate form (31) is that the overlap integral in Eq. 290 is Gaussian and can be easily evaluated. For four vortices forming a square with a side of length $d$ one obtains

$$
F_{1234}=\frac{1}{2 \pi^{2} \xi^{2}} e^{-d^{2} / \pi \xi^{2}}
$$

For $d \gtrsim \xi$ one again expects a crossover to a simple exponential behavior $\sim e^{-d / \xi}$.

To complete the estimate we need the characteristic value of $V_{0}$. Assuming screened Coulomb interaction between electrons of the form $V_{\mathrm{TF}}(\boldsymbol{r})=\left(e^{2} / r\right) e^{-r / \lambda}$ with $\lambda$ the Thomas-Fermi screening length, we find $V_{0}=$ $\int d^{2} r V_{\mathrm{TF}}(\boldsymbol{r})=2 \pi e^{2} \lambda$. Putting everything together we thus arrive at an estimate

$$
g_{\square} \simeq-\frac{2 e^{2} \lambda}{\pi \xi^{2}} e^{-d^{2} / \pi \xi^{2}} .
$$

A more transparent expression arises if we introduce the Bohr radius $a_{0}=\hbar^{2} / m e^{2} \simeq 0.52 \times 10^{-10} \mathrm{~m}$ and the associated energy scale $\epsilon_{0}=e^{2} / 2 a_{0} \simeq 13.6 \mathrm{eV}$,

$$
g_{\square} \simeq-\epsilon_{0} \frac{4}{\pi} \frac{a_{0} \lambda}{\xi^{2}} e^{-d^{2} / \pi \xi^{2}} .
$$


a

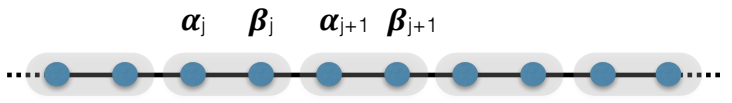

b

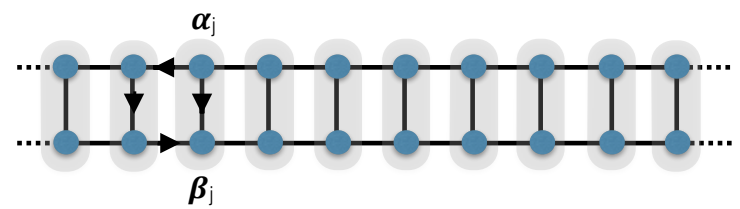

c

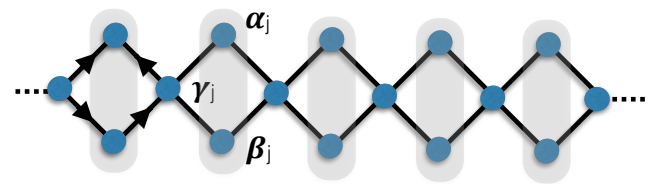

d

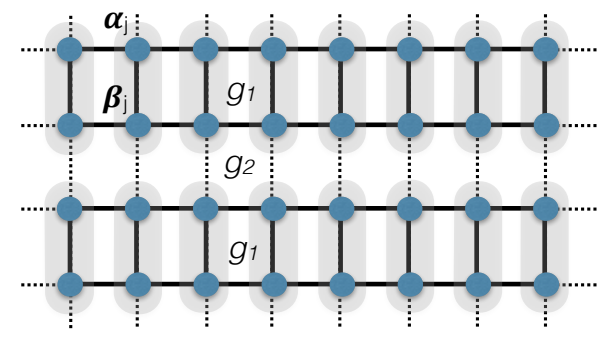

e

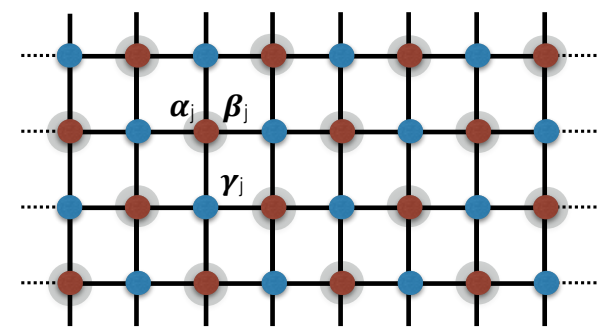

FIG. 2: Lattice structures for models with strongly interacting Majorana fermions. a Simple 1D chain, b two-leg ladder and c the diamond chain. d Simple square lattice with two types of plaquettes characterized by interaction strength $g_{1}$ and $g_{2}$ and e the modified square lattice with alternate sites (rendered in red) occupied by double vortices. The arrows in panels $\mathbf{b}$ and $\mathbf{c}$ indicate our choice of the $\mathrm{Z}_{2}$ gauge factors for the Majorana hopping terms consistent with Eqs. (14) and (15).

To estimate the typical interaction strength we take the experimentally measured [23] coherence length $\xi \simeq 29$ $\mathrm{nm}$. The value of the screening length $\lambda$ in this system is not known but we note that it should be significantly longer than the screening inside a typical metal (or a superconductor) because in the setup of Ref. 23 the surface layer of the STI is separated from the SC substrate by the insulating bulk of the STI crystal with thickness $h \simeq 3-10 \mathrm{~nm}$. The STI surface state itself should not screen efficiently because of its low density of states. A simple exercise in elementary electrostatics shows that the screening length in this situation is then bound from below by distance $h$. This can be seen, for instance, by noting that the screening field can be attributed to the relevant image charge placed distance $h$ below the SC surface. We can thus use distance $h$ as a rough estimate for the screening length $\lambda \simeq 10 \mathrm{~nm}$ to obtain an estimate for the interaction strength $g_{\square} \simeq(10.6 \mathrm{meV}) \times e^{-d^{2} / \pi \xi^{2}}$. An even stronger interaction could be achieved in a material with a shorter coherence length or longer Thomas-Fermi screening length $\lambda$. Because of the exponential dependence on the intervortex distance $d$, the interaction effects will be most pronounced when $d$ does not exceed $\xi$ by a wide margin. For instance when $d=2 \xi$ we obtain a respectable $g_{\square} \approx 3 \mathrm{meV}$ interaction scale.

The interaction strength is to be compared with the direct hopping amplitude, which under the same assumptions as above becomes

$$
t_{12} \simeq \mu e^{-d^{2} / 4 \pi \xi^{2}} .
$$

Strong correlation regime obtains when $\mu$ is tuned such that $\left|t_{12}\right| \ll\left|g_{\square}\right|$. In a typical experiment $\mu$ is controled by a combination of chemical doping and electrostatic gating. The latter is a continuous process in which, pre- sumably, the average $\mu$ can be tuned as close to zero as desired. From this perspective, achieving the interaction dominated regime should not present a significan problem, except of course that one must also ensure that the interaction effects are not obscured by disorder. We further discuss disorder effects in Sec. IV.C.

\section{LATTICE MODELS WITH INTERACTING MAJORANA FERMIONS}

We now proceed to study specific interacting models in one and two spatial dimensions. We focus on lattice geometries whose building blocks are either 1D line segments or square plaquettes because they most naturally accommodate the four-fermion interaction terms (16). We begin with $1 \mathrm{D}$ structures which can be physically realized by inducing $\mathrm{SC}$ order in a narrow strip on the surface of an STI and then applying magnetic field of appropriate strength perpendicular to the surface. In 2D we focus on vortex lattices with square symmetry. We note that although in most conventional superconductors natural vortex lattices are triangular [42, there exist materials with a strong four-fold anisotropy in which square vortex lattices have been experimentally observed [55]58. To engineer more complex vortex structures one could also employ various techniques that generate vortex pinning [59 61]. This involves, essentially, perturbing the superconductor in a controlled fashion on the nanoscale to create a pattern of regions with locally suppressed SC order parameter $\Delta(\boldsymbol{r})$. Such regions then attract and pin vortex cores due to the lower condensation energy. With sufficiently strong pinning one can, in principle, create almost arbitrary arrangement of vortices, includ- 
ing systems with e.g. multiply quantized vortices which are otherwise energetically unstable.

\section{A. One-dimensional lattice models}

One may expect on symmetry grounds that onedimensional vortex lattice structures will arise when a strip of a superconducting thin-film material is deposited on the STI surface and subjected to a perpendicular magnetic field. Theoretical calculations within the GinzburgLandau theory indeed predict a single line of vortices forming along the long axis of the strip at low fields and more complicated structures with multiple lines at higher fields 62. Some of these predictions have been confirmed experimentally 63. Importantly, these calculations also indicate that the intervortex distance in such configurations is typically much smaller than the distance between vortices and the strip edge. This means that the interactions between Majorana zero modes bound to vortices will dominate over any residual interactions with lowenergy Dirac fermions present in the ungapped surface of the STI (note also that the density of states of the latter vanishes when $\mu \approx 0$ ).

\section{Linear chain}

A simple linear chain depicted in Fig. 2a is described by an interacting Hamiltonian of the form

$$
\mathcal{H}_{\text {int }}=g_{1} \sum_{j} \alpha_{j} \beta_{j} \alpha_{j+1} \beta_{j+1}+g_{2} \sum_{j} \beta_{j} \alpha_{j+1} \beta_{j+1} \alpha_{j+2} .
$$

Here $\alpha_{j}$ and $\beta_{j}$ denote two Majoranas in the two site unit cell $j$. In a uniform chain $g_{1}=g_{2}$ but we consider here a more general case of dimerized bond lengths leading to alternating couplings $g_{1}$ and $g_{2}$. The Hamiltonian (36) can be brought to a more familiar form by performing a Wigner-Jordan transformation suitable for Majorana fermions [54] to spin variables $\boldsymbol{\sigma}_{j}$,

$$
\alpha_{j}=\left(\prod_{k=1}^{j-1} \sigma_{k}^{x}\right) \sigma_{j}^{z}, \quad \beta_{j}=i\left(\prod_{k=1}^{j-1} \sigma_{k}^{x}\right) \sigma_{j}^{z} \sigma_{j}^{x} .
$$

One obtains

$$
\mathcal{H}_{\mathrm{int}}=-g_{1} \sum_{j} \sigma_{j}^{x} \sigma_{j+1}^{x}-g_{2} \sum_{j} \sigma_{j}^{z} \sigma_{j+2}^{z}
$$

an interesting variant of the XY model, with nearest neighbor spin interactions along $x$ and next nearest interactions along $z$. This is an example of a spin model that would not naturally arise in a system where fundamental degrees of freedom are electron spins. Yet it emerges here from a very simple and natural structure composed of interacting Majorana fermions.
Adding direct hopping terms (assuming again a dimerized lattice) described by

$$
\mathcal{H}_{\mathrm{kin}}=i t_{1} \sum_{j} \alpha_{j} \beta_{j}+i t_{2} \sum_{j} \beta_{j} \alpha_{j+1}
$$

gives, in the spin representation,

$$
\mathcal{H}_{\text {kin }}=-t_{1} \sum_{j} \sigma_{j}^{x}-t_{2} \sum_{j} \sigma_{j}^{z} \sigma_{j+1}^{z}
$$

The full Hamiltonian $\mathcal{H}=\mathcal{H}_{\text {int }}+\mathcal{H}_{\text {kin }}$ is not exactly solvable for a general set of parameters but has several special points in the parameter space where exact solutions are known. These include an anisotropic XY model for $g_{2}=t_{1}=0$ and a transverse field Ising model when $g_{1}=t_{2}=0$ or when $g_{1}=g_{2}=0$. A detailed exploration of the phase diagram of this model is beyond the scope of this study and we leave it to future work. It is clear, however, that the model exhibits a rich phase diagram with gapped and gapless phases, some of which are topologically non-trivial and carry unpaired Majorana zero modes at the edges.

\section{Two-leg ladder}

Next we consider a two-leg ladder shown in Fig. 2 b. The interacting Hamiltonian is given by the first term in Eq. (36). This model is exactly solvable for an arbitrary interaction strength $g$ on the square plaquette. To see this note that each four-fermion term commutes with the Hamiltonian and is therefore a constant of motion. Furthermore, adding hopping $t$ along the rung does not spoil the model's integrability, although hopping $t^{\prime}$ along the legs does. After the WJ transformation (37) the Hamiltonian can be written as $\mathcal{H}=\mathcal{H}_{0}+\mathcal{H}^{\prime}$ with

$$
\begin{aligned}
\mathcal{H}_{0} & =-g \sum_{j} \sigma_{j}^{x} \sigma_{j+1}^{x}-t \sum_{j} \sigma_{j}^{x} \\
\mathcal{H}^{\prime} & =-t^{\prime} \sum_{j}\left(\sigma_{j}^{y} \sigma_{j+1}^{z}-\sigma_{j}^{z} \sigma_{j+1}^{y}\right) .
\end{aligned}
$$

The signs of $t$ and $t^{\prime}$ terms here reflect the $\mathrm{Z}_{2}$ gauge factors indicated in Eq. (15). At the neutrality point $\left(t=t^{\prime}=0\right)$ and assuming $g>0$ the ground state is a doubly degenerate ferromagnet. In the fermion language this corresponds to complex fermions $c_{j}=\frac{1}{2}\left(\alpha_{j}+i \beta_{j}\right)$ on each rung either all occupied or all empty. Turning on $t \neq 0$ removes the two-fold degeneracy. Since this is a gapped state one expects it to remain stable against the perturbation $\mathcal{H}^{\prime}$ as long as $t^{\prime}$ remains weak. 


\section{Diamond chain}

As the final 1D example we consider a diamond chain depicted in Fig. 2r. The interacting Hamiltonian is

$$
\mathcal{H}_{\text {int }}=g_{1} \sum_{j \text { odd }} \gamma_{j} \alpha_{j} \beta_{j} \gamma_{j+1}+g_{2} \sum_{j \text { odd }} \gamma_{j+1} \alpha_{j+1} \beta_{j+1} \gamma_{j+2},
$$

where once again we allow for the possibility of dimerization. We observe that products $\alpha_{j} \beta_{j}$ commute with $\mathcal{H}_{\text {int }}$ and with one another. They can thus be replaced by classical variables $i s_{j}= \pm i$. The Hamiltonian becomes

$$
\mathcal{H}_{\text {int }}=i \sum_{j \text { odd }}\left(g_{1} s_{j} \gamma_{j} \gamma_{j+1}+g_{2} s_{j+1} \gamma_{j+1} \gamma_{j+2}\right)
$$

describing a simple $1 \mathrm{D}$ chain with hoppings $g_{1}$ and $g_{2}$ between nearest neighbor sites. Because there are no closed loops in such a linear chain we can adopt a gauge in which $s_{j}=1$ for all $j$. The Hamiltonian (44) then coincides with the Kitaev chain model [53. Accordingly, its spectrum is gapped whenever $g_{1} \neq g_{2}$. For an open ended chain with sites labeled $j=1 \ldots 2 N$ the phase with $g_{1}<g_{2}$ is topological and has unpaired Majorana zero modes bound to its two ends while $g_{1}>g_{2}$ corresponds to the trivial phase. $g_{1}=g_{2}$ marks the critical point separating the two phases. Adding $\mathcal{H}_{\text {kin }}$ to the interacting Hamiltonian (43) spoils its integrability but again we may expect the gapped phases to be robust against small detuning from the neutrality point.

\section{B. Two-dimensional lattice models}

We now turn to 2D lattice geometries. A simple square lattice depicted in Fig. $2 \mathrm{~d}$ is not exactly solvable and we shall discuss its phase diagram below. We consider first a modified square lattice shown in Fig. 2 e which represents a somewhat artificial but exactly solvable $2 \mathrm{D}$ geometry with strong interactions. It is obtained by populating one sublattice with doubly quantized vortices each containing two exact Majorana zero modes $\alpha_{\boldsymbol{j}}, \beta_{\boldsymbol{j}}$. The dominant interaction terms in this arrangement are of the form

$$
\mathcal{H}_{\text {int }}=g \sum_{\boldsymbol{j}, \boldsymbol{\nu}} \alpha_{\boldsymbol{j}} \beta_{\boldsymbol{j}} \alpha_{\boldsymbol{j}+\boldsymbol{\nu}} \beta_{\boldsymbol{j}+\boldsymbol{\nu}}+g^{\prime} \sum_{\boldsymbol{j}, \boldsymbol{\delta}_{1}, \boldsymbol{\delta}_{2}} \alpha_{\boldsymbol{j}} \beta_{\boldsymbol{j}} \gamma_{\boldsymbol{j}+\boldsymbol{\delta}_{1}} \gamma_{\boldsymbol{j}+\boldsymbol{\delta}_{2}}
$$

where $\boldsymbol{\delta}= \pm \hat{x}, \pm \hat{y}$ are the nearest neighbor vectors while $\boldsymbol{\nu}$ second neighbor vectors on the square lattice. The model is solvable because once again products $\alpha_{\boldsymbol{j}} \beta_{\boldsymbol{j}}$ commute with $\mathcal{H}_{\text {int }}$ (and with one another) and can thus be replaced by classical variables $i s_{j}= \pm i$. The resulting Hamiltonian is bilinear in the $\gamma$ operators residing on the single vortex sites and can be analyzed in a straightforward fashion. Depending on the relative sign and amplitude of the couplings $g$ and $g^{\prime}$ various phases are possible, including a gapless metallic phase when $g \gg g^{\prime}>0$ and, interestingly, dispersionless flat band at zero energy when $g<0$ and $|g| \gg\left|g^{\prime}\right|$. A detailed discussion of this model is given in Appendix $\mathrm{B}$.

A simple square lattice model depicted in Fig. 2d cannot be reduced to a non-interacting problem and we study it by a combination of approximate analytical techniques and by exact numerical diagonalization on small clusters. To facilitate the discussion we consider a dimerized situation with couplings $g_{1}$ and $g_{2}$ on alternating rows of plaquettes, described by

$\mathcal{H}_{\text {int }}=g_{1} \sum_{\boldsymbol{j}} \alpha_{\boldsymbol{j}} \beta_{\boldsymbol{j}} \alpha_{\boldsymbol{j}+\boldsymbol{x}} \beta_{\boldsymbol{j}+\boldsymbol{x}}+g_{2} \sum_{\boldsymbol{j}} \beta_{\boldsymbol{j}} \alpha_{\boldsymbol{j}-\boldsymbol{y}} \beta_{\boldsymbol{j}+\boldsymbol{x}} \alpha_{\boldsymbol{j}+\boldsymbol{x}-\boldsymbol{y}}$

In the limit $g_{2}=0$ the system breaks up into a collection of two-leg ladders already discussed above. Assuming $g_{1}, g_{2} \geq 0$ the exact ground state is a direct product of the ground states of the individual ladders. In the language of Ising spins defined in Eq. 37) these are doubly degenerate 1D ferromagnets. The ground state thus exhibits a $2^{N_{y}}$-fold degeneracy, where $N_{y}$ is the number of unit cells in the $y$ direction. The spectrum of excitations is gapped and the lowest excited state at energy $2 g_{1}$ has one of the spins reversed. Inclusion of nonzero $g_{2}$ can be seen to suppress the ferromagnetic order in the individual ladders by promoting excitations. A reasonable conjecture is that the gapped phase persist all the way to the isotropic point $g_{2}=g_{1}$ which marks a quantum phase transition to another gapped state that is adiabatically connected to a set of independent ladders that occur at $g_{1}=0$.

We have performed a standard mean-field (MF) analysis by decoupling $\mathcal{H}_{\text {int }}$ in all possible channels involving Majorana bilinears on nearest and next nearest neighbor bonds. At $g_{2}=0$ this procedure yields the exact ground state with $\Delta_{1}=g_{1}\left\langle i \alpha_{\boldsymbol{j}} \beta_{\boldsymbol{j}}\right\rangle= \pm g_{1}$ and all other order parameters zero. The two possible signs correspond to two degenerate ferromagnetic ground states on each ladder. Interestingly, this solution persists as the meanfield ground state for all values of $g_{2}<g_{1}$. At $g_{2}=g_{1}$ the MF theory predicts a strong first order transition to a state characterized by non-vanishing order parameter $\Delta_{2}=g_{2}\left\langle i \beta_{\boldsymbol{j}} \alpha_{\boldsymbol{j}-\boldsymbol{y}}\right\rangle= \pm g_{2}$ which then persists all the way to $g_{1}=0$ where it becomes the exact ground state of $\mathcal{H}_{\text {int }}$. To ascertain the accuracy of the MF solution we carried out exact numerical diagonalizations (ED) of $\mathcal{H}_{\text {int }}$ for a system containing $N_{x} \times 4$ lattice sites with $N_{x}$ up to 19 (see Appendix C). Some representative results are displayed in Fig. 3. These indicate that MF treatment provides a reasonable approximation for $g_{2} / g_{1} \ll 1$ but breaks down when the two couplings are comparable. Specifically, ED indicates a continuous phase transition at $g_{2}=g_{1}$ with the gap closing smoothly at that point.

We expect the gapped phases of the $2 \mathrm{D}$ model to remain robust against small detuning from the neutrality point. However, at the criticality, such detuning is likely to drive the system into another phase, adiabatically connected to the noninteracting system of Majorana fermions described by Hamiltonian (14). Our conjectured 

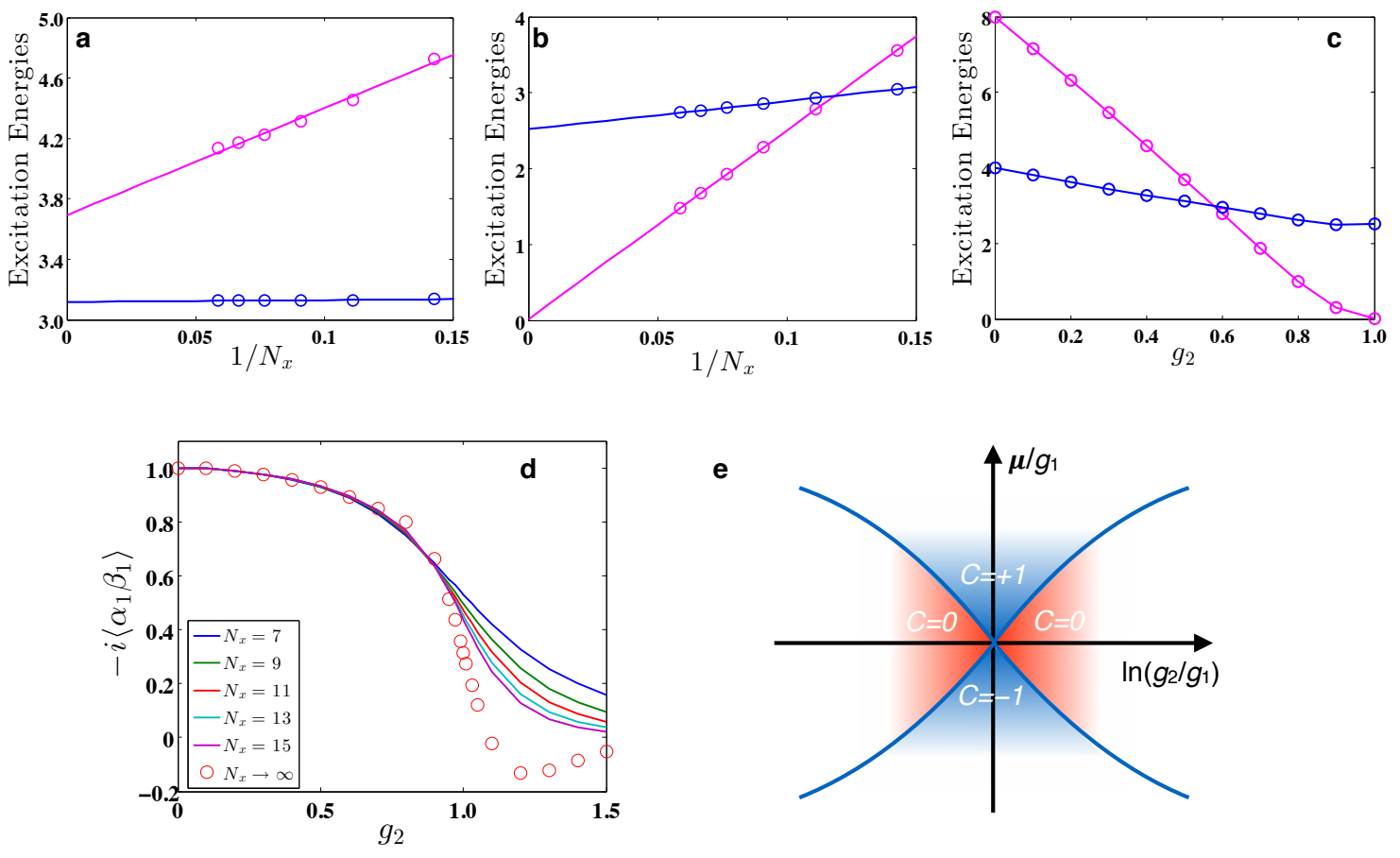

FIG. 3: Interacting system of Majorana fermions on the simple square lattice. Panels a-c show the finite size scaling analysis of the many-body excitation energies of the system obtained by exact numerical diagonalization. Details of the numerical procedure are described in Appendix C Energies of the two lowest excited states are plotted as a function of $1 / N_{x}$ for $g_{2}=0.5$ in $\mathbf{a}$ and $g_{2}=1.0 \mathrm{in} \mathbf{b}$. The excitation energies extrapolated to $N_{x} \rightarrow \infty$ are displayed in panel $\mathbf{c}$ as a function of $g_{2}$. This plot shows that the gap closes at $g_{2}=g_{1}$ indicating a phase transition. We note that the first excited state here exhibits degeneracy that grows with a system size. Panel $\mathbf{d}$ shows the order parameter $\Delta_{1}=-i\left\langle\alpha_{1} \beta_{1}\right\rangle$ as a function of $g_{2}$ for various system sizes. The infinite system extrapolation is obtained by assuming $\Delta_{1} \simeq c_{0}+c_{1} / N_{x}+c_{2} / N_{x}^{2}$. The order parameter goes to zero continuously at $g_{2}=g_{1}$ supporting the notion of the continuous phase transition. In panels a-c $g_{1}=1.0$ is held constant. e The schematic phase diagram for the simple square lattice system.

phase diagram describing this situation is displayed in Fig. 33. The gapped phases in the interaction dominated regime are separated from the hopping dominated phases by topological phase transitions. This can be seen by analyzing the noninteracting Hamiltonian (14). It describes spinless fermions with charge conjugation symmetry. Since the time reversal symmetry is absent the system is in topological class D which has integer classification in $d=2$. Assuming that $t_{i j}$ is dominated by first and second neighbor hoppings $t$ and $t^{\prime}$ the system is gapped and one can easily calculate the corresponding Chern number $C=\operatorname{sgn}\left(t^{2} t^{\prime}\right)= \pm 1$. Recalling furthermore that for small chemical potential $t, t^{\prime} \propto \mu$, as shown in Sec. II, we obtain $C=\operatorname{sgn}(\mu)$, leading to the phase diagram illustrated in Fig. 33. The interaction dominated phases by contrast are adiabatically connected to systems of decoupled two leg ladders and are thus topologically trivial with $C=0$.

\section{Physical feasibility and proposed experimental observations}

Models discussed in this Section can be engineered in a laboratory provided that several conditions are met. The key requirement is the ability to tune the chemical potential $\mu$ of the STI surface state to the close vicinity of the neutrality point. Although the most common STIs in the $\mathrm{Bi}_{2} \mathrm{Se}_{3}$ family do not naturally grow in this regime, neutrality point can be reached in these via chemical doping and by electrostatic gating in the thin film or flake geometry. Remarkably, tantalizing evidence for intrinsic surface superconductivity with $T_{c} \simeq 9 \mathrm{~K}$ and $\Delta_{0} \simeq 5$ $\mathrm{meV}$ has recently been reported [16] in topological insulator $\mathrm{Sb}_{2} \mathrm{Te}_{3}$ whose growth chemistry has been tuned to achieve neutrality. Although the mechanism behind the emergence of superconducting order in this material is presently not known, if confirmed this system could form an ideal platform for the exploration of the lattice models with interacting Majorana fermions. In other, more recently discovered STI materials, such as the ternary $\mathrm{Bi}_{2} \mathrm{Te}_{2} \mathrm{Se}$, the $\mu \approx 0$ condition naturally obtains in a stoichiometric crystal 64, 65. Quaternary compounds $\mathrm{Bi}_{2-x} \mathrm{Sb}_{x} \mathrm{Te}_{3-y} \mathrm{Se}_{y}$ can in turn be robustly tuned into 
their neutrality point 66].

The samples must also be sufficiently clean so that the interaction effects are not obscured by disorder. The situation here resembles fractional quantum Hall systems where the sample quality is of paramount importance. Disorder that breaks the chiral symmetry of the Fu-Kane model, such as the fluctuating scalar potential, will generate random Majorana hopping between the adjacent vortices. These must be negligible compared to the interaction scale $g$ that we estimated to be of the order of several meV. Disorder that does not break the symmetry, such as irregularities in the vortex positions or fluctuations in the SC pairing amplitude, will not generate hopping terms but will introduce a random component $\delta g$ in the interaction strengths. Understanding the effect of disorder in a strongly interacting system is a difficult problem, one that lies beyond the scope of this study. By thinking about those interacting models that are exactly solvable (such as the two leg ladder and the diamond chain) we may conclude that weak disorder $|\delta g| \ll|g|$ will have negligible effect on the gapped phases but could affect the nature of the critical points in some cases. In models that are not integrable disorder could lead to more interesting phenomena such as the many-body localization. This, obviously, is a potentially interesting topic for future studies.

The most obvious experimental tool to probe the interacting systems we described in this study is scanning tunneling microscopy (STM). This technique is uniquely suited to image vortex lattices at the nanoscale [58] as well as to detect bound states present in the vortex cores 67, 68. A first step towards observing the complex phenomena associated with interactions will be to resolve a single Majorana zero mode in the vortex core of the FuKane model and its splitting as a result of hybridization with another zero mode localized in a nearby vortex. We note that once a suitable sample with $\mu \approx 0$ has been fabricated this should be a relatively easy task because in this limit Fu-Kane model predicts a single vortex core state at zero energy separated from all other core states by a gap whose amplitude is close to the full SC gap $\Delta_{0}$ 69, 70]. With the SC gap of the order of meV, as seen in Ref. 23, a state of the art STM should have no problem clearly resolving the zero modes and their splitting due to hybridization or interaction effects.

Once the zero modes are detected the next step will consist of establishing the effect of interactions in small clusters of vortices. This again, should be relatively straightforward. Interaction effects are easy to distinguish from simple hybridization because they require four or more vortices to occur. Thus, a smoking gun test for the interaction effect is to probe the zero mode splitting in a group of 2, 3 and 4 vortices. Hybridization, if present, will split the zero modes in all cases while interaction will only cause splitting in the last case. When the interaction effect is confirmed in such small clusters then one can move onto larger lattices which will, for correct geometries, show interesting collective phenomena.
We have discussed in this Section some specific examples of vortex lattice geometries that lead to simple interacting models with Majorana fermions. Even these basic structures display interesting behaviors. The actual experimental vortex lattice geometries will depend on the details of the physical samples and we shall not attempt here to specify the precise conditions for the formation of a given structure. Instead, we note that since STM can be used to map out both the lattice structure and the electronic state of vortices, theory will work best in conjunction with experiment to unveil the physics of strong interactions in these systems.

\section{OUTLOOK}

When the chemical potential is tuned to coincide with the Dirac point in the superconducting surface of a strong topological insulator Majorana fermions bound to the vortex cores show a completely flat band, protected by the chiral symmetry. In this regime the nature of the ground state is determined by interactions between the Majorana zero modes and the system must be regarded as inherently strongly correlated. We gave examples of lattice geometries in one and two dimensions for which the ground state of the strongly interacting system can be found exactly. In other cases, such as the simple 1D Majorana chain, exact solution of the interacting problem is unknown but the Hamiltonian maps onto an interesting spin problem which can be studied by standard techniques such as the density matrix renormalization group (DMRG). Although well understood theoretically spin models in 1D often face significant hurdles when it comes to their experimental realizations. For instance the fine details of quantum criticality in the transverse field Ising model - perhaps the most widely studied 1D spin model - have been only recently mapped out experimentally 71. Our construction may thus enable new experimental realizations of these well studied models. In addition, it may help realize spin models that do not naturally occur in systems whose fundamental degrees of freedom are spins, as in the case of the interacting 1D Majorana chain.

Interesting phenomena occur also in two-dimensional systems. The simple square lattice shows an intriguing phase diagram with both topological and trivial gapped phases as well as a quantum phase transition that cannot be described by mean field theory. Further interesting phases in 2D may arise in lattices with triangular symmetry which we have not considered in this study.

Physical realizations of interacting systems with Majorana fermions in some respects similar to ours have been previously discussed in the context of semiconductor quantum wire networks [43 45]. The existence of Majorana fermions in the individual quantum wires has been established by recent ground breaking experiments 46 52 . However, assembling these into large arrays with uniform properties and tunable interaction and hopping 
parameters appears to be a much more difficult challenge, one that will likely require new experimental methodologies. By contrast, scaling the systems of few vortices with Majorana zero modes, such as those observed in $\mathrm{Bi}_{2} \mathrm{Te}_{3} / \mathrm{NbSe}_{2}$ heterostructures [23, to large lattices required in our proposal seems to be rather straightforward. The key issue that must be surmounted to achieve the strong correlation regime here is the ability to tune the system to its global neutrality point. In addition, local fluctuations of the chemical potential must remain sufficiently small as to render disorder effects negligible compared to the interaction energy scale. We estimated in Sec. III that the characteristic interaction energy in $\mathrm{Bi}_{2} \mathrm{Te}_{3} / \mathrm{NbSe}_{2}$ heterostructures is $\sim 10 \mathrm{meV}$. We emphasize that only disorder strength averaged over distances comparable to intervortex spacing $d$ (of the order of $10-100 \mathrm{~nm}$ ) must be small compared to the interaction energy, which should be achievable in clean STI samples.

The ultimate goal of these constructions is to find novel phases that cannot be adiabatically deformed into phases of weakly interacting fermions or interesting phase transitions that do not have a free particle description. That such phases or transitions can indeed occur in these systems could be anticipated because Majorana interactions of the form Eq. (16) play a pivotal role in the construction of various "interaction enabled" topological phases introduced in the seminal work by Fidkowski and Kitaev [54. Our work indicates how such interactions can be generated and controlled in a system that is now physically accessible thanks to the recent experimental breakthroughs [15-21, 23. We note that recently a specific model has been formulated by Lapa, Teo and Hughes 72 that produces an interaction enabled topological crystalline phase (which has no analog in a weakly interacting system) and also employs Majorana interaction of the type discussed in this work as the key component. One can show that such a phase can be in fact constructed from the ingredients introduced in this study [73].

\section{ACKNOWLEDGMENT}

The authors thank I. Affleck, J. Alicea, T. Liu, A. Rahmani, G. Refael, K. Shtengel and X. Zhu for useful discussions. The authors are indebted to NSERC, CIfAR and Max Planck - UBC Centre for Quantum Materials for support. M.F. acknowledges The Aspen Center for Physics and IQMI at Caltech for hospitality during various stages of this project.

\section{Appendix A: Phase factors, branch cuts and the $Z_{2}$ gauge structure}

In this Appendix we outline the computation of the relevant phase factors that enter the overlap integrals for Majorana zero modes in Eq. (13) and the interaction am- plitudes 25]. We also explain how the $\mathrm{Z}_{2}$ gauge factors that appear in the Majorana tight binding model arise from branch cuts present in the vortex lattice.

Although the method outlined here is applicable to an arbitrary arrangement of vortices, we focus, for the sake of concreteness, on a periodic vortex lattice such as the one depicted in Fig. 4. Following [29] we define the phase $\theta_{j}$ that enters the definition of the Majorana wavefunction (7) at a point $\boldsymbol{r}_{j}$ immediately to the right of the given vortex center, to avoid the phase singularity. The overlap integral between the two vortices at $\boldsymbol{r}_{i}$ and $\boldsymbol{r}_{j}$ is then given, according to Eq. (18), as $t_{i j}=\mu F_{i j} \sin \omega_{i j}$ with

$$
\omega_{i j}=\int_{\boldsymbol{r}_{i}}^{\boldsymbol{r}_{j}}\left(\frac{1}{2} \nabla \theta-\frac{e}{\hbar c} \boldsymbol{A}\right) \cdot d \mathbf{l},
$$

where we have restored $\hbar$. The integrand in Eq. A1, which we henceforth call $\boldsymbol{\Omega}$, is closely related to the superfluid velocity 42 .

$$
\boldsymbol{v}_{s}=\frac{\hbar}{m^{*}}\left(\nabla \theta-\frac{e^{*}}{\hbar c} \boldsymbol{A}\right)=\frac{2 \hbar}{m^{*}} \boldsymbol{\Omega} .
$$

Here $e^{*}=2 e$ and $m^{*}$ are, respectively, the effective charge and mass of the Cooper pair. The superfluid velocity distribution in the vortex lattice can be calculated in a straightforward way 42 which we review below for completeness. It is related to the supercurrent $\boldsymbol{j}_{s}=e^{*} n_{s} \boldsymbol{v}_{s}$ where $n_{s}$ represents the superfluid density.

The calculation proceeds by taking the curl of $\boldsymbol{j}_{s}$,

$$
\nabla \times \boldsymbol{j}_{s}=n_{s} \frac{e^{*} \hbar}{m^{*}}\left(\nabla \times \nabla \theta-\frac{e^{*}}{\hbar c} \boldsymbol{B}\right),
$$

and noting that

$$
\nabla \times \nabla \theta=2 \pi \hat{z} \sum_{j} \delta\left(\boldsymbol{r}-\boldsymbol{r}_{j}\right)
$$

where $\boldsymbol{r}_{j}$ are the vortex positions and we are assuming that the SC interface lies in the $x-y$ plane. We now use the Ampère's law $\nabla \times \boldsymbol{B}=(4 \pi / c) \boldsymbol{j}_{s}$ to eliminate the current from Eq. A3). We thus find the London equation for $\boldsymbol{B}$ in the vortex lattice,

$$
\boldsymbol{B}-\lambda_{L}^{2} \nabla^{2} \boldsymbol{B}=\frac{1}{2} \Phi_{0} \hat{z} \sum_{j} \delta\left(\boldsymbol{r}-\boldsymbol{r}_{j}\right),
$$

where $\lambda_{L}^{2}=m c^{2} / 4 \pi e^{* 2} n_{s}$ is the London penetration depth and $\Phi_{0}=h c / e$ the flux quantum. For a periodic lattice the equation can be solved by Fourier transforming,

$$
\boldsymbol{B}(\boldsymbol{r})=\frac{1}{2} \Phi_{0} \hat{z} \sum_{\boldsymbol{G}} \frac{e^{i \boldsymbol{G} \cdot \boldsymbol{r}}}{1+\lambda_{L}^{2} G^{2}}
$$

where the sum extends over all reciprocal vectors $\boldsymbol{G}$ of the vortex lattice. From the knowledge of $\boldsymbol{B}$ one can 

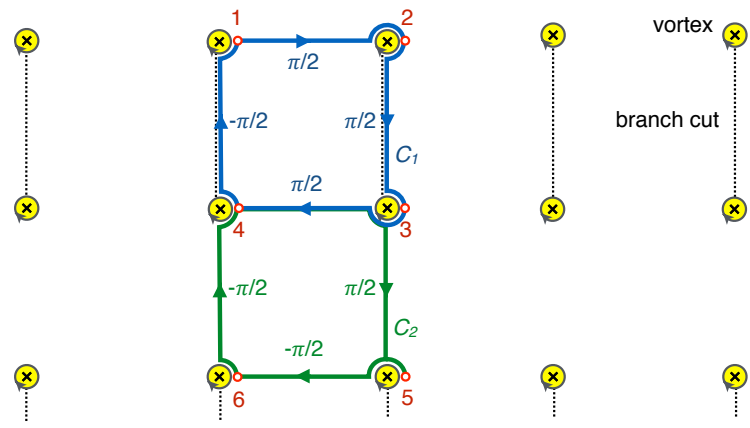

FIG. 4: Phase factors and branch cuts in a square vortex lattice. Oriented solid lines indicate integration paths between the reference points located just to the right of each each vortex center. Dashed lines represent a specific choice of the branch cuts discussed in the text.

reconstruct the supercurrent via Eq. A3 and from it $\boldsymbol{v}_{s}$. Finally,

$$
\boldsymbol{\Omega}(\boldsymbol{r})=\pi \sum_{\boldsymbol{G}} \frac{i \boldsymbol{G} \times \hat{z}}{\lambda_{L}^{-2}+G^{2}} e^{i \boldsymbol{G} \cdot \boldsymbol{r}} .
$$

The gauge invariant phase factors $\omega_{i j}$ can now be determined by a straightforward integration of $\boldsymbol{\Omega}(\boldsymbol{r})$ indicated in Eq. A1.

The above method works for any vortex lattice but in cases with high symmetry, such as the square lattice, the phase factors can be deduced without performing a detailed calculation. Consider the lattice depicted in Fig. 4. The integration paths between points $\boldsymbol{r}_{j}$ have been chosen to consist of straight line segments and circular segments. The latter are needed to avoid the phase singularities located at each vortex center. In the following we think of these as having an arbitrarily small radius so that the contribution to the line integral along the cirgular segment comes exclusively from the adjacent singularity. Now consider the path $C_{1}$ indicated in Fig. 4. The corresponding line integral $\oint_{C_{1}} \boldsymbol{\Omega} \cdot d \mathbf{l}=\int(\nabla \times \boldsymbol{\Omega}) \cdot d \boldsymbol{S}$ can be seen to equal to $\pi$; it encloses two vortices, each contributing flux $\pi$ and a half quantum of magnetic flux in the opposite direction contributing $-\pi$. We furthermore note that counting just the contribution of the circular line segments around the vortices one gets the same answer $\pi$ for the total flux. We are thus led to a conclusion that the straight line segments do not contribute to $\omega_{i j}$. This same conclusion can be reached by similarly examining the path $C_{2}$ which contains total flux $-\pi$. Thus, in the square vortex lattice, we can determine the phase factors $\omega_{i j}$ by simply adding up the contributions from the circular segments around the individual vortices, which are given by their angular length divided by two. This leads to the phase factors $\omega_{i j}= \pm \pi / 2$ indicated in the Figure, a result that can be confirmed by an explicit calculation using Eq. (A7).

The above arguments contain an important subtlety that has to do with branch cuts. Consider for instance the path indicated in Fig. 4 between points 1 and 2. Had we chosen a path avoiding the vortex from below (instead of going above it) we would have found the phase to be $-\pi / 2$. More generally, $\omega_{i j}$ changes to $\omega_{i j} \pm \pi$, depending on which way we decide to avoid the singularity. The magnitude of $t_{i j}$ is independent of this choice but its sign depends on it because $\sin \left(\omega_{i j} \pm \pi\right)=-\sin \omega_{i j}$. This is the origin of the $Z_{2}$ gauge structure in Eq. (14). The latter is inherent to the tight binding models with Majorana fermions and arises here from the physics of branch cuts. In order to consistently determine the signs of $t_{i j}$, which become physically relevant when there exist closed loops in the model, one must define $\omega_{i j}$ in a globally unique fashion. This can be done by specifying branch cuts across which $\boldsymbol{\Omega}(\boldsymbol{r})$ changes discontinuously. A branch cut emanates from each vortex core and can be chosen to terminate in another vortex core. An example of a specific choice of branch cuts is given in Fig. 4. Integration paths that do not intersect any branch cuts then furnish a globally consistent definition of the gauge invariant phase factors $\omega_{i j}$. Different choices of branch cuts correspond to different $\mathrm{Z}_{2}$ gauges for Majorana fermions, but they leave the physical observables unchanged. The phase factors indicated in Fig. 4 have been obtained in accord with this prescription. They define a periodic lattice with two vortices per unit cell and are consistent with the Grosfeld-Stern rule Eq. (15). The same phase factors are used for the computation of the interaction amplitudes in Sec. III.

\section{Appendix B: Exactly solvable 2D model}

The building block for the solvable Majorana model in $2 \mathrm{D}$ is a doubly quantized vortex defined by Eq. (3) with $(n=2)$. The solution for the Majorana wavefunction goes along similar lines as for the single vortex 34. We search for zero mode solutions of operator $D$ defined in Eq. (6) with $n=2$ in the form

$$
\chi_{m}(\mathbf{r})=\frac{1}{\sqrt{2}}\left(\begin{array}{c}
e^{i((1-m) \varphi+\theta / 2-\pi / 4)} u_{m}(r) \\
e^{-i(m \varphi+\theta / 2-\pi / 4)} v_{m}(r)
\end{array}\right) .
$$

We substitute this into $D$ to obtain

$$
\left\{\begin{array}{c}
\Delta_{0}(r) u_{m}(r)+\left(\partial_{r}-\frac{m}{r}\right) v_{m}(r)=0 \\
\Delta_{0}(r) v_{m}(r)+\left(\partial_{r}-\frac{1-m}{r}\right) u_{m}(r)=0 .
\end{array}\right.
$$

It is known 34 that these equations have normalizable real solutions for $m=0,1$, for which it holds

$$
u_{1}=v_{0}, v_{1}=u_{0}
$$

This observation allows us to write the field operator of the zero modes

$$
\begin{aligned}
\alpha(\boldsymbol{r}) & \propto\left[e^{i(\varphi+\theta / 2-\pi / 4)} c_{\boldsymbol{r} \downarrow}+e^{-i(\varphi+\theta / 2-\pi / 4)} c_{\boldsymbol{r} \downarrow}^{\dagger}\right] u_{0}(r) \\
& +\left[e^{i(\theta / 2-\pi / 4)} c_{\boldsymbol{r} \downarrow}+e^{-i(\theta / 2-\pi / 4)} c_{\boldsymbol{r} \downarrow}^{\dagger}\right] v_{0}(r), \quad(\mathrm{B} 4) \\
\beta(\boldsymbol{r}) & \propto i\left[e^{i(\varphi+\theta / 2-\pi / 4)} c_{\boldsymbol{r} \downarrow}-e^{-i(\varphi+\theta / 2-\pi / 4)} c_{\boldsymbol{r} \downarrow}^{\dagger}\right] u_{0}(r) \\
& -i\left[e^{i(\theta / 2-\pi / 4)} c_{\boldsymbol{r} \downarrow}-e^{-i(\theta / 2-\pi / 4)} c_{\boldsymbol{r} \downarrow}^{\dagger}\right] v_{0}(r) .
\end{aligned}
$$


a)

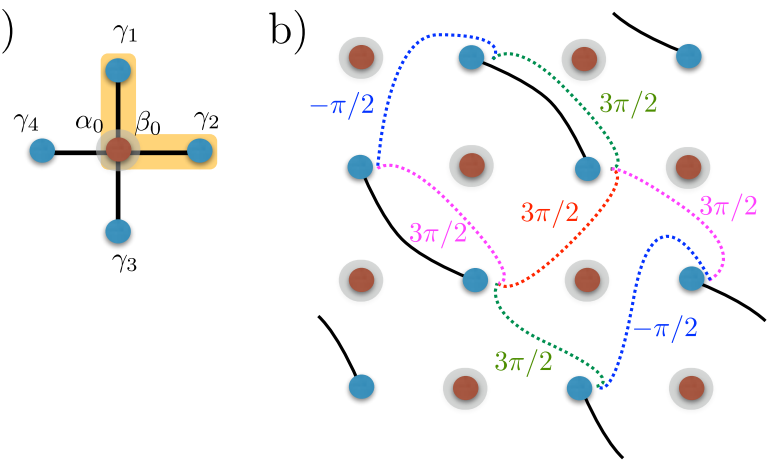

FIG. 5: Modified square lattice structure a A site with the doubly quantized vortex surrounded by singly quantized vortices. The dominant type of interaction within such a node is shaded in yellow. b Phase difference structure for a choice of gauge in the modified square lattice.

It is easy to show that the density is then given by

$$
\rho_{\alpha \beta} \propto\left[u_{0}^{2}(r)-v_{0}^{2}(r)\right] .
$$

This expression depends only on the distance from the vortex core. It decays exponentially on distances longer than the coherence length $\xi$.

We are now interested in the dominant interactions between the Majoranas in such a model. For this we notice that the interaction is the largest for the combinations $g \alpha_{\boldsymbol{j}} \beta_{\boldsymbol{j}} \alpha_{\boldsymbol{j}+\boldsymbol{\nu}} \beta_{\boldsymbol{j}+\boldsymbol{\nu}}$ and $g^{\prime} \alpha_{\boldsymbol{j}} \beta_{\boldsymbol{j}} \gamma_{\boldsymbol{j}+\boldsymbol{\delta}_{1}} \gamma_{\boldsymbol{j}+\boldsymbol{\delta}_{2}}$ depending on how strong the screening of the Coulomb interactions is. The corresponding interaction strengths are proportional to $\exp \left[-\left|\mathbf{R}_{\boldsymbol{j}+\boldsymbol{\nu}}-\mathbf{R}_{\boldsymbol{j}}\right| / R_{c}\right]$, where $R_{c}$ is the Coulomb screening length, and $\exp \left[-\left(\left|\mathbf{R}_{\boldsymbol{j}}-\mathbf{R}_{\boldsymbol{j}+\boldsymbol{\delta}_{1}}\right|+\left|\mathbf{R}_{\boldsymbol{j}}-\mathbf{R}_{\boldsymbol{j}+\boldsymbol{\delta}_{\boldsymbol{2}}}\right|\right) / \xi\right]$.

Consider first the case $R_{c}<\xi$. The dominant interaction is the $g^{\prime}$ term as Coulomb interaction on long lengthscale decays faster than the overlap of the Majorana wavefunctions. This interaction term dominates as it does not involve the smallness due to the screening of the Coulomb interaction, only due to the decay of the Majorana wavefunctions. Following the observation of the previous section that $\rho_{i j} \propto \sin \left(\left(\theta_{i}-\theta_{j}\right) / 2\right)$, we see that the interaction is proportional to $\sin \left(\left(\theta_{\boldsymbol{j}+\boldsymbol{\delta}_{1}}-\theta_{\boldsymbol{j}+\boldsymbol{\delta}_{2}}\right) / 2\right)$. This is the interaction of the form $\rho_{\alpha \beta} \rho_{\gamma \gamma}$. The rest of the terms in (27) are canceling each other, since $\alpha_{\boldsymbol{j}}$ and $\beta_{\boldsymbol{j}}$ are different by as if they had a phase difference $\pi$.

As we noted in the main text, the products $i \alpha_{\boldsymbol{j}} \beta_{\boldsymbol{j}}$ commute with the Hamiltonian and with each other. Thus they are conserved quantities $s_{j}= \pm 1$ signaling the occupation of the Andreev states $c_{\boldsymbol{j}}=\frac{1}{2}\left(\alpha_{\boldsymbol{j}}+i \beta_{\boldsymbol{j}}\right)$. This means that we can trace out these degrees of freedom from the model and obtain the hopping amplitudes between the single-vortex sites. The relevant phase differences $\theta$ are depicted in Fig. 5 5 . We consider for illustration two possible configurations of $s_{j}$ : ferromagnetic (all +1 or all -1 ) and antiferromagnetic (staggered on the two sublattices). It is easy to see that for the FM configuration the hopping amplitudes on a given bond contributed by the two adjacent double vortex sites add up while for the AF configuration they cancel. Therefore, in the AF case the resulting hopping model produces a completely flat Majorana band. Meanwhile for the FM configuration the hopping model will be of the form indicated in Eq. (5) of the main text with the nearest neighbor hopping $t=2 g^{\prime}$. The energy spectrum then consists of a pair of dispersing bands with energies

$$
E_{\boldsymbol{k}}= \pm 4 g^{\prime} \sqrt{\sin ^{2}\left(\frac{k_{x}+k_{y}}{2}\right)+\sin ^{2}\left(\frac{k_{x}-k_{y}}{2}\right)}
$$

where $\boldsymbol{k}$ ranges over the reduced Brillouin zone. Occupying the negative energy states in Eq. (B6) clearly produces lower ground state energy than occupying a flat band at zero energy, therefore hinting that the FM state is the ground state of the system. To prove this we should also consider all other possible occupations of the lattice. Our numerics in the systems up to $6 \times 6$ unit cells shows that the FM state is the stable ground state of the system. There is no reason this should change in larger systems. For the screened Coulomb interaction, therefore, a gapless metallic phase with the excitation spectrum (B6) is produced.

Now consider the case $R_{c}>\xi$. Here the dominant interaction is between the double vortices. If the interaction is the usual Coulomb repulsion, $g \gg g^{\prime}>0$, then the preferred occupation $s_{\boldsymbol{j}}$ of the double vortices is antiferromagnetic and the hopping model obtained is the flat Majorana band, as discussed above. Smaller terms involving four single vortex sites can split this degeneracy, but the model thus obtained is not integrable. If the interaction is attractive, $g<0,|g| \gg\left|g^{\prime}\right|$, then the preferred occupation of the double vortices is ferromagnetic and the resulting model is the same as for the screened Coulomb, a gapless dispersing Majorana band Eq. B6].

\section{Appendix C: 2D single Majorana vortex lattice}

The exact diagonalization study of the system on the simple square lattice is performed by transforming the Hamiltonian 46 to the fermionic basis, $\alpha_{\boldsymbol{j}}=c_{\boldsymbol{j}}^{\dagger}+c_{\boldsymbol{j}}$, $\beta_{\boldsymbol{j}}=i\left(c_{\boldsymbol{j}}^{\dagger}-c_{\boldsymbol{j}}\right)$. The Hamiltonian then becomes

$$
\begin{aligned}
\mathcal{H}_{\text {int }} & =-g_{1} \sum_{\boldsymbol{j}}\left(2 N_{\boldsymbol{j}}-1\right)\left(2 N_{\boldsymbol{j}+x}-1\right) \\
& +g_{2} \sum_{\boldsymbol{j}}\left(c_{\boldsymbol{j}}^{\dagger}-c_{\boldsymbol{j}}\right)\left(c_{\boldsymbol{j}+x}^{\dagger}-c_{\boldsymbol{j}+x}\right) \\
& \times\left(c_{\boldsymbol{j}-y}^{\dagger}+c_{\boldsymbol{j}-y}\right)\left(c_{\boldsymbol{j}-y+x}^{\dagger}+c_{\boldsymbol{j}-y+x}\right)
\end{aligned}
$$

where $N_{\boldsymbol{j}}=c_{\boldsymbol{j}}^{\dagger} c_{\boldsymbol{j}}$ denotes the number operator and $\boldsymbol{j}$ indicates the $2 \mathrm{D}$ coordinate $(n, m)$ of the unit cell. If we were to directly diagonalize the many-body Hamiltonian, only a small system can be numerically treated. Fortunately, $\mathcal{H}_{\text {int }}$ can be block-diagonalized by defining the 
fermion parity operators

$$
\hat{F}_{n}^{x}=(-1)^{\sum_{m} N_{n, m}}, \quad \hat{F}_{m}^{y}=(-1)^{\sum_{n} N_{n, m}},
$$

which commute with the Hamiltonian $\mathcal{H}_{\text {int }}$ and among themselves. Their eigenvalues $( \pm 1)$ are good quantum numbers and label the different blocks of the Hamiltonian. However, these operators are not independent since they are connected by the total fermionic parity operator $\hat{F}=\prod_{n} \hat{F}_{n}^{x}=\prod_{m} \hat{F}_{m}^{y}$.

We consider separately the cases when the number $N_{x}$ of unit cells in the $x$ direction is odd and even. The Hamiltonian can be easily block-diagonalized by $F_{n}^{x}= \pm 1$ and $F_{m}^{y}= \pm 1$. We are able to numerically solve the block-diagonalized Hamiltonian for a system containing $N_{x} \times 4$ unit cells with $N_{x}$ up to 19 as follows. We first find one of the degenerate ground states $|G\rangle$ in the parity sector $F_{n}^{x}=1$ and $F_{m}^{y}=1$ for all $n$ and $m$. We then use the operator $\hat{A}_{\tilde{m}}=\prod_{n} \alpha_{n, \tilde{m}}$ to generate the remaining ground states. Note that $\hat{A}_{\tilde{m}}$ commutes with $\mathcal{H}_{\text {int }}$ but anticommutes with all $\hat{F}_{\tilde{n}}^{x}$. When it acts on a ground state it thus flips the sign of all $F_{\tilde{n}}^{x}$ generating a new ground state in a different parity sector. When we subsequently apply $\hat{A}_{\tilde{m}^{\prime}}$ with $\tilde{m}^{\prime} \neq \tilde{m}$ to this new ground state all $F_{\tilde{n}}^{x}$ flip back. This construction indicates that there exist at least two degenerate ground states. For even $N_{x}$, our numerical results support the two-fold ground state degeneracy. For odd $N_{x}, \hat{A}_{\tilde{m}}$ also flips the sign of $F_{\tilde{m}}^{y}$. Since the number of $\hat{F}_{\tilde{m}}^{y}$ operators is $N_{y}$ and $F_{\tilde{m}}^{y}= \pm 1$ the degenerate ground states are given by

$$
\left|F_{\tilde{m}_{ \pm}}^{y}= \pm 1\right\rangle=\prod_{\tilde{m}_{-}} \hat{A}_{\tilde{m}_{-}}|G\rangle .
$$

It follows that the number of the degenerate ground states is at least $2^{N_{y}}$. This agrees with the degeneracy that occurs in the extreme anisotropy limit $g_{2}=0$, already discussed in the main text.

Interestingly, the systems with even and odd $N_{x}$ exhibit different physical properties even in the thermodynamic limit. When $N_{x}$ is even, by performing a $Z_{2}$ gauge transformation $\alpha_{2 l, m} \rightarrow-\alpha_{2 l, m}$, the Hamiltonian $\mathcal{H}_{\text {int }}$ changes the sign. That is, when the many-body state has energy $E$, the state after the gauge transformation has energy $-E$. This many-body version of the particle-hole symmetry shows that $g_{1}, g_{2} \geq 0$ describes identical physics as $g_{1}, g_{2} \leq 0$. However, for odd $N_{x}$, $\alpha_{2 l, m} \rightarrow-\alpha_{2 l, m}$ does not simply flip the sign of $\mathcal{H}_{\text {int }}$ due to the frustration at the boundary with the periodic boundary condition. Hence, systems with positive $g_{1}$ and $g_{2}$ are different from those with negative $g_{1}$ and $g_{2}$ in this case.

After obtaining the many-body wavefunctions of the ground states from the exact diagonalization, the order parameter $\Delta_{1}=-i\left\langle\alpha_{\boldsymbol{j}} \beta_{\boldsymbol{j}}\right\rangle$ can be computed as a ground state expectation value in different parity sectors. We mainly focus on odd $N_{x}$. Because $\hat{A}_{\tilde{m}}=\prod_{n} \alpha_{n, \tilde{m}}$ connects the ground states in the different parity sectors, $\Delta_{1}$ must be computed in only one of the parity sectors, say $F_{\tilde{n}}^{x}=1$ and $F_{\tilde{m}}^{y}=1$ for all $\tilde{n}$ and $\tilde{m}$, as shown in Fig. $3 \mathrm{~d}$. The expectation value flips the sign when we consider the ground state with parity $F_{m}^{y}=-1$.
[1] D.C. Tsui, H.L. Stormer, and A.C. Gossard, Phys. Rev. Lett. 48, 1559 (1982).

[2] R.B. Laughlin, Phys. Rev. Lett. 50, 1395 (1983).

[3] E. Tang, J.-W. Mei and X.-G. Wen, Phys. Rev. Lett. 106, 236802 (2011).

[4] K. Sun, Z. Gu, H. Katsura and S. Das Sarma, Phys. Rev. Lett. 106, 236803 (2011).

[5] T. Neupert, L. Santos, C. Chamon and C. Mudry, Phys. Rev. Lett. 106, 236804 (2011).

[6] X. Hu, M. Kargarian and G. Fiete, Phys. Rev. B 84 155116 (2011).

[7] D.N. Sheng, Z.-C. Gu, K. Sun, and L. Sheng, Nat. Comm. 2, 389 (2011).

[8] N. Regnault, B.A. Bernevig, Phys. Rev. X 1, 021014 (2011).

[9] Nigel R. Cooper and Jean Dalibard Phys. Rev. Lett. 110 185301 (2013).

[10] N. Y. Yao, A. V. Gorshkov, C. R. Laumann, A. M. Lauchli, J. Ye, and M. D. Lukin Phys. Rev. Lett. 110 $185302(2013)$

[11] J. Alicea, Rep. Prog. Phys. 75, 076501 (2012).

[12] C.W.J. Beenakker, Annu. Rev. Con. Mat. Phys. 4, 113 (2013).
[13] T. D. Stanescu and S. Tewari, J. Phys.: Condens. Matter 25, 233201 (2013).

[14] S.R. Elliott, and M. Franz, Rev. Mod. Phys. 87, 137 (2015).

[15] G. Koren, T. Kirzhner, E. Lahoud, K. B. Chashka, and A. Kanigel, Phys. Rev. B 84, 224521 (2011).

[16] L. Zhao, H. Deng, I. Korzhovska, J. Secor, M. Begliarbekov, Z. Chen, E. Andrade, E. Rosenthal, A. Pasupathy, V. Oganesyan, and L. Krusin-Elbaum, arXiv:1408.1046.

[17] B. Sacépé, J. B. Oostinga, J. Li, A. Ubaldini, N. J. G. Couto, E. Giannini, and A. F. Morpurgo, Nat. Comm. 2, 575 (2011).

[18] F. Qu, F. Yang, J. Shen, Y. Ding, J. Chen, Z. Ji, G. Liu, J. Fan, X. Jing, C. Yang, and Li Lu, Scientific Reports 2, 339 (2012).

[19] J. R. Williams, A. J. Bestwick, P. Gallagher, S. S. Hong, Y. Cui, A. S. Bleich, J. G. Analytis, I. R. Fisher, and D. Goldhaber-Gordon, Phys. Rev. Lett. 109, 056803 (2012).

[20] S. Cho, B. Dellabetta, A. Yang, J. Schneeloch, Z. Xu, T. Valla, G. Gu, M. J. Gilbert, and N. Mason, Nat. Comm. 4, 1689 (2013).

[21] S.-Y. Xu, N. Alidoust, I. Belopolski, A. Richardella, C. Liu, M. Neupane, G. Bian, S.-H. Huang, R. 
Sankar, C. Fang, B. Dellabetta, W. Dai, Q. Li, M. J. Gilbert, F. Chou, N. Samarth, and M. Zahid Hasan, arXiv: 1410.5405

[22] L. Fu and C. L. Kane, Phys. Rev. Lett. 100, 096407 (2008).

[23] J.-P. Xu, C. Liu, M.-X. Wang, J. Ge, Z.-L. Liu, X. Yang, Y. Chen, Y. Liu, Z.-A. Xu, C.-L. Gao, D. Qian, F.C. Zhang, and J.-F. Jia, Phys. Rev. Lett. 112, 217001 (2014).

[24] M. Cheng, R. M. Lutchyn, V. Galitski, and S. Das Sarma, Phys. Rev. Lett. 103, 107001 (2009).

[25] M. Cheng, R. M. Lutchyn, V. Galitski, and S. Das Sarma, Phys. Rev. B 82, 094504 (2010).

[26] Y. E. Kraus and A. Stern, New J. Phys. 13105006 (2011).

[27] J. Zhou, Y.-J. Wu, R.-W. Li, J. He, and S.-P. Kou, Europhys. Lett. 102, 47005 (2013).

[28] M. A. Silaev, Phys. Rev. B 88, 064514 (2013).

[29] R. R. Biswas, Phys. Rev. Lett. 111, 136401 (2013).

[30] H.-H. Hung, P. Ghaemi, T. L. Hughes, and M. J. Gilbert, Phys. Rev. B 87, 035401 (2013)

[31] J.C.Y. Teo and C.L. Kane, Phys. Rev. B 82, 115120 (2010).

[32] N. Read and D. Green, Phys. Rev. B 61, 10267 (2000).

[33] D.A. Ivanov, Phys. Rev. Lett. 86, 268 (2001).

[34] R. Jackiw and P. Rossi, Nucl. Phys. B 190, 681 (1981).

[35] E.J. Weinberg, Phys. Rev. D 24, 2669 (1981).

[36] E. Grosfeld and A. Stern, Phys. Rev. B 73, 201303(R) (2006).

[37] V. Lahtinen, A.W.W. Ludwig, J.K. Pachos, and S. Trebst, Phys. Rev. B 86, 075115 (2012).

[38] V. Lahtinen, A.W.W. Ludwig, and S. Trebst, Phys. Rev. B 89, 085121 (2014).

[39] V. Shivamoggi, G. Refael, and J.E. Moore, Phys. Rev. B 82, 041405(R) (2010).

[40] C.R. Laumann, A.W.W. Ludwig, D.A. Huse, and S. Trebst, Phys. Rev. B 85, 161301(R) (2012).

[41] C.R. Laumann, D.A. Huse, A.W.W. Ludwig, G. Refael, S. Trebst, and M. Troyer, Phys. Rev. B 85, 224201 (2012).

[42] M. Tinkham, Introduction to Superconductivity (McGraw Hill, New York) 1996.

[43] F. Hassler and D. Schuricht, New J. Phys. 14, 125018 (2012).

[44] B.M. Terhal, F. Hassler, and D.P. DiVincenzo, Phys. Rev. Lett. 108, 260504 (2012).

[45] G. Kells, V. Lahtinen, and J. Vala, Phys. Rev. B 89, 075122 (2014).

[46] V. Mourik, K. Zuo, S. M. Frolov, S. R. Plissard, E. P. A. M. Bakkers, and L. P. Kouwenhoven, Science 336, 1003 (2012).

[47] A. Das, Y. Ronen, Y. Most, Y. Oreg, M. Heiblum, and H. Shtrikman, Nature Physics 8, 887 (2012).

[48] M. T. Deng, C. L. Yu, G. Y. Huang, M. Larsson, P.
Caroff, and H. Q. Xu, Nano Letters 12, 6414 (2012).

[49] L. P. Rokhinson, X. Liu, and J. K. Furdyna, Nat. Phys. 8, 795 (2012).

[50] A. D. K. Finck, D. J. Van Harlingen, P. K. Mohseni, K. Jung, and X. Li, Phys. Rev. Lett. 110, 126406 (2013).

[51] H. O. H. Churchill, et al., Phys. Rev. B 87, 241401 (2013).

[52] E. J. H. Lee, X. Jiang, M. Houzet, R. Aguado, C. M. Lieber, and S. D. Franceschi, Nature Nanotechnology, 9, $79,(2014)$.

[53] A.Y. Kitaev, Phys. Usp. 44, 131 (2001).

[54] L. Fidkowski and A. Kitaev, Phys. Rev. B 81, 134509 (2010).

[55] T. M. Riseman, et al., Nature (London) 396, 242 (1998).

[56] R. Gilardi et al. Phys. Rev. Lett. 88, 217003 (2002).

[57] P. J. Curran, V. V. Khotkevych, S. J. Bending, A. S. Gibbs, S. L. Lee, and A. P. Mackenzie, Phys. Rev. B 84, 104507 (2011).

[58] B.B. Zhou, S. Misra, E.H. da Silva Neto,P. Aynajian, R. E. Baumbach, J. D. Thompson, E.D. Bauer and A. Yazdani, Nature Phys. 9, 474 (2013).

[59] O. Daldini, P. Martinoli, J. L. Olsen, and G. Berner, Phys. Rev. Lett. 32, 218 (1974).

[60] M. Baert, V. V. Metlushko, R. Jonckheere, V. V. Moshchalkov, and Y. Bruynseraede, Phys. Rev. Lett. 74, 3269 (1995).

[61] K. Harada, O. Kamimura, H. Kasai, T. Matsuda, A. Tonomura, and V.V. Moshchalkov, Science 274, 1167 (1996)

[62] G. Teniers, L.F. Chibotaru, A. Ceulemans and V.V. Moshchalkov, Europhys. Lett. 63, 296 (2003).

[63] M. Morelle, J. Bekaert, and V.V. Moshchalkov, Phys. Rev. B 70, 094503 (2004).

[64] Z. Ren, A.A. Taskin, S. Sasaki, K. Segawa, and Y. Ando, Phys. Rev. B 82, 241306(R) (2010).

[65] J. Xiong, A. C. Petersen, Dongxia Qu, R. J. Cava, N. P. Ong, Physica E 44, 917 (2012).

[66] Z. Ren, A. A. Taskin, Sa. Sasaki, K. Segawa, Y. Ando, Phys. Rev. B 84, 165311 (2011).

[67] F. Gygi and M. Schluter, Phys. Rev. B 43, 7609 (1991).

[68] I. Maggio-Aprile et al., Phys. Rev. Lett. 75, 2754 (1995).

[69] A. M. Cook, M. M. Vazifeh, M. Franz, Phys. Rev. B 86, 155431 (2012).

[70] Ref. 69 considers Majorana zero mode at the end of a thin topological insulator wire. The calculation for a vortex in a 2D STI surface is similar and leads to the same result regarding the higher energy bound states.

[71] R. Coldea, D. A. Tennant, E. M. Wheeler, E. Wawrzynska, D. Prabhakaran, M. Telling, K. Habicht, P. Smeibidl, and K. Kiefer, Science 327, 177 (2010).

[72] M.F. Lapa, J.C.Y. Teo and T.L. Hughes, arXiv:1409.1234

[73] C.-K. Chiu, D.I. Pikulin, and M. Franz, arXiv:1502.03432 